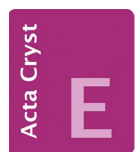

CRYSTALLOGRAPHIC COMMUNICATIONS

ISSN 2056-9890

\section{Crystal structure of bis[(oxalato- $\left.\kappa^{2} O^{1}, O^{2}\right)(1,4,8,11$ - tetraazacyclotetradecane- $\left.\kappa^{4} N\right)$ chromium(III)] dichromate octahydrate from synchrotron X-ray data}

\author{
Dohyun Moon $^{\mathrm{a}}$ and Jong-Ha Choi ${ }^{\mathrm{b} *}$
}

Received 13 February 2017

Accepted 14 February 2017

Edited by J. Simpson, University of Otago, New Zealand

Keywords: crystal structure; cyclam; chromium(III) complex; bidentate oxalato ligand; cis$\checkmark$ conformation; dichromate anion; hydrogen bonding; synchrotron radiation.

CCDC reference: 1532785

Supporting information: this article has supporting information at journals.iucr.org/e

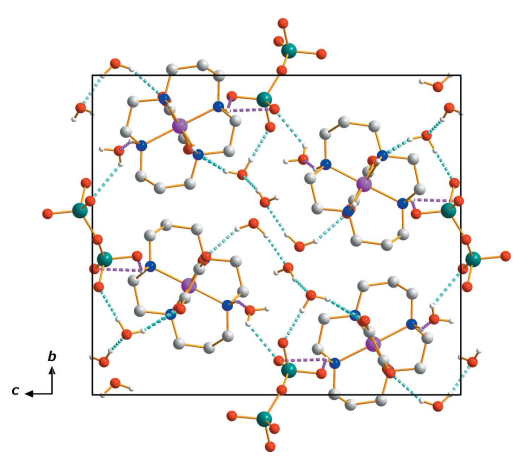

OPEN $\odot$ ACCESS
aPohang Accelerator Laboratory, POSTECH, Pohang 37673, Republic of Korea, and ${ }^{\mathbf{b}}$ Department of Chemistry, Andong National University, Andong 36729, Republic of Korea. ${ }^{*}$ Correspondence e-mail: jhchoi@anu.ac.kr

The asymmetric unit of the title compound, $\left[\mathrm{Cr}\left(\mathrm{C}_{2} \mathrm{O}_{4}\right)\left(\mathrm{C}_{10} \mathrm{H}_{24} \mathrm{~N}_{4}\right)\right]_{2}\left[\mathrm{Cr}_{2} \mathrm{O}_{7}\right] \cdot$ $8 \mathrm{H}_{2} \mathrm{O}\left(\mathrm{C}_{10} \mathrm{H}_{24} \mathrm{~N}_{4}=1,4,8,11\right.$-tetraazacyclotetradecane, cyclam; $\mathrm{C}_{2} \mathrm{O}_{4}=$ oxalate, ox) contains one $[\mathrm{Cr}(\mathrm{ox})(\mathrm{cyclam})]^{+}$cation, one half of a dichromate anion that lies about an inversion centre so that the bridging $\mathrm{O}$ atom is equally disordered over two positions, and four water molecules. The terminal $\mathrm{O}$ atoms of the dichromate anion are also disordered over two positions with a refined occupancy ratio 0.586 (6):0.414 (6). The $\mathrm{Cr}^{\mathrm{III}}$ ion is coordinated by the four $\mathrm{N}$ atoms of the cyclam ligand and one bidentate oxalato ligand in a cis arrangement, resulting in a distorted octahedral geometry. The $\mathrm{Cr}-\mathrm{N}$ (cyclam) bond lengths are in the range 2.069 (2)-2.086 (2) $\AA$, while the average $\mathrm{Cr}-$ $\mathrm{O}(\mathrm{ox})$ bond length is $1.936 \AA$. The macrocyclic cyclam moiety adopts the cis- $\mathrm{V}$ conformation. The dichromate anion has a staggered conformation. The crystal structure is stabilized by intermolecular hydrogen bonds involving the cyclam $\mathrm{N}-\mathrm{H}$ groups and water $\mathrm{O}-\mathrm{H}$ groups as donors, and the $\mathrm{O}$ atoms of oxalate ligand, water molecules and the $\mathrm{Cr}_{2} \mathrm{O}_{7}{ }^{2-}$ anion as acceptors, giving rise to a three-dimensional network.

\section{Chemical context}

Chromium $(\mathrm{Cr})$ is considered a trace element essential for the proper functioning of living organisms and is also a highly toxic material (Yusof \& Malek, 2009). Cr can exist in all oxidation states from 0 to VI, the most common oxidation states in water being $\mathrm{Cr}^{\mathrm{III}}$ and $\mathrm{Cr}^{\mathrm{VI}}$. In an aqueous environment, the toxicity of $\mathrm{Cr}^{\mathrm{VI}}$ has been shown to be greater than that of $\mathrm{Cr}^{\mathrm{III}}$ (Guzel et al., 2016). Transition metal complexes of the cyclam (1,4,8,11-tetraazacyclotetradecane, $\left.\mathrm{C}_{10} \mathrm{H}_{24} \mathrm{~N}_{4}\right)$ ligand have been the subject of numerous investigations because of their particular conformational stereochemistry (Poon \& Pun, 1980; Choi, 2009; Subhan et al., 2011). Recently, it has been found that cyclam derivatives and their metal complexes exhibit anti-HIV activity (Ronconi \& Sadler, 2007; De Clercq, 2010; Ross et al., 2012). The conformation of the macrocyclic ligand is a very important factor for co-receptor recognition. Therefore, knowledge of the conformation and hydrogen-bonding interactions in $\mathrm{Cr}^{\mathrm{III}}-\mathrm{Cr}^{\mathrm{VI}}$ complex systems containing the cyclam ligand has become important in the development of new anti-HIV drugs (De Clercq, 2010). The use of such complexes for the more effective removal of toxic metals is also important (Guzel et al., 2016). As part of a study of the conformation and structure of (cyclam)chromium(III) complexes with auxiliary ligand(s) and various anions, we 
report here the structural characterization of the new complex salt, $\left[\mathrm{Cr}\left(\mathrm{C}_{2} \mathrm{O}_{4}\right)\left(\mathrm{C}_{10} \mathrm{H}_{24} \mathrm{~N}_{4}\right)\right]_{2}\left[\mathrm{Cr}_{2} \mathrm{O}_{7}\right] \cdot 8 \mathrm{H}_{2} \mathrm{O}$, (I).

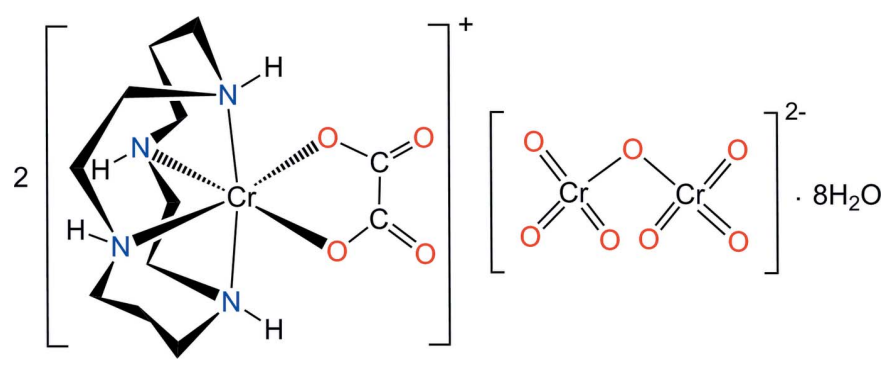

\section{Structural commentary}

An ellipsoid plot of the molecular components in (I) is shown in Fig. 1 along with the atom-numbering scheme. The structure is another example of a $[\mathrm{Cr}(\mathrm{ox})(\mathrm{cyclam})]^{+}$cation (Choi et al., 2004b; Moon \& Choi, 2016b), but with a different counteranion. The asymmetric unit contains one $[\mathrm{Cr}(\mathrm{ox})(\text { cyclam })]^{+}$ cation, one half of a $\mathrm{Cr}_{2} \mathrm{O}_{7}{ }^{2-}$ anion (completed by inversion symmetry with the bridging $\mathrm{O}$ atom disordered about the inversion centre) and four non-coordinating water molecules. The three terminal $\mathrm{O}$ atoms of the dichromate anion are also disordered over two positions with occupancy ratio of the atom pairs $\mathrm{O} 2 B 1 / \mathrm{O} 2 B 2, \mathrm{O} 3 B 1 / \mathrm{O} 3 B 2$ and $\mathrm{O} 4 B 1 / \mathrm{O} 4 B 2$ converging at $0.586(6): 0.414(6)$. The conformation of the cyclam ligand can be described as cis-V (anti-anti) (Subhan et al., 2011). In the complex cation, the $\mathrm{Cr}^{\mathrm{III}}$ ion is coordinated by the four nitrogen atoms of the cyclam ligand in a folded conformation. Two oxygen atoms of the oxalato ligand complete the distorted octahedral coordination sphere. The $\mathrm{Cr}-\mathrm{N}$ bond lengths from the donor atoms of cyclam ligand lie in the range 2.069 (2) to 2.086 (2) $\AA$, in good agreement with those determined in cis- $\left[\mathrm{Cr}\left(\mathrm{N}_{3}\right)_{2}(\right.$ cyclam $\left.)\right] \mathrm{ClO}_{4}[2.069(3)-$ 2.103 (3) $\AA$ ] (Meyer et al., 1998), cis-[Cr(ONO $)_{2}($ cyclam $\left.)\right] \mathrm{NO}_{2}$ [2.0874 (16)-2.0916 (15) А] (Choi et al., 2004a), [Cr(acac)(cyclam $)]\left(\mathrm{ClO}_{4}\right)_{2} \cdot 0.5 \mathrm{H}_{2} \mathrm{O}[2.070(5)-2.089(5) \AA]($ acac $=$ acetylacetonate; Subhan et al., 2011), cis-[Cr(NCS $)_{2}($ cyclam $\left.)\right] \mathrm{NCS}$ [2.0851 (14)-2.0897 (14) ̊] (Moon et al., 2013) and $\left[\mathrm{CrCl}_{2^{-}}\right.$ (cyclam) $][\mathrm{Cr}($ ox $)($ cyclam $)]\left(\mathrm{ClO}_{4}\right)_{2} \quad[2.075$ (5)-2.096 (5) $\AA$ (Moon \& Choi, 2016b). However, the $\mathrm{Cr}-\mathrm{N}$ bond lengths of the cyclam ligand with a cis geometry are slightly longer than those found in trans-[Cr(NCS $)_{2}($ cyclam $) \mathrm{ClO}_{4} \quad[2.046(2)-$ 2.060 (2) $\AA$ ] (Friesen et al., 1997), trans-[Cr(ONO) $)($ cyclam) $] \mathrm{BF}_{4}[2.064(4)-2.073(4) \AA]$ (De Leo et al., 2000), trans $-\left[\mathrm{Cr}\left(\mathrm{NH}_{3}\right)_{2}\right.$ (cyclam) $]\left[\mathrm{ZnCl}_{4}\right] \mathrm{Cl} \cdot \mathrm{H}_{2} \mathrm{O} \quad[2.0501$ (15)$2.0615(15) \AA]$ (Moon \& Choi, 2016a) and trans-[Cr(nic$\mathrm{O})_{2}($ cyclam $\left.)\right] \mathrm{ClO}_{4}[2.058$ (4)-2.064 (4) $\AA$ ] (nic-O = O-coordinated nicotinate; Choi, 2009).

The $\mathrm{Cr} 1 A-\mathrm{O} 1 A$ distance $[1.9665(16) \AA]$ in the oxalate ligand is very slightly longer than the $\mathrm{Cr} 1 A-\mathrm{O} 3 A$ [1.9600 (16) $\AA$ ] bond length. This elongation may be attributed to the weak hydrogen bond formed by $\mathrm{O} 1 A\left(x,-y+\frac{1}{2}\right.$, $z-\frac{1}{2}$ ) with the $\mathrm{O} 3 \mathrm{~S}-\mathrm{H} 2 \mathrm{O} 3$ atoms of a water molecule. The mean $\mathrm{Cr}-\mathrm{O}$ bond length is comparable to the mean values of $1.959,1.956$ and $1.969 \AA$ observed in $[\mathrm{Cr}(\mathrm{ox})($ cyclam $)] \mathrm{ClO}_{4}$

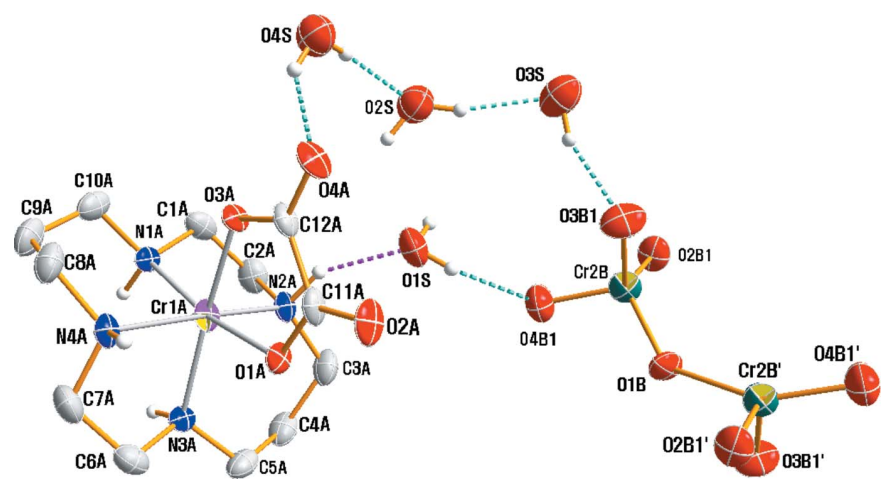

Figure 1

A perspective view of the asymmetric unit of the title of compound, (I), with the dichromate anion, which lies about an inversion centre, drawn in full. Displacement ellipsoids are drawn at the $30 \%$ probability level and primed atoms are related by the symmetry operation $(2-x,-y, 1-z)$. For clarity, only the major disorder components are shown for the disordered dichromate anion.

(Choi et al., 2004b), $\left[\mathrm{CrCl}_{2}\right.$ (cyclam) $][\mathrm{Cr}($ ox $)($ cyclam $)]\left(\mathrm{ClO}_{4}\right)_{2}$ (Moon \& Choi, 2016b) and $\mathrm{K}_{3}\left[\mathrm{Cr}(\mathrm{ox})_{3}\right] \cdot 3 \mathrm{H}_{2} \mathrm{O}$ (Taylor, 1978), respectively. The five- and six-membered chelate rings of the cyclam ligand adopt gauche and stable chair conformations, respectively. As expected for a bidentate ox ligand, the $\mathrm{O} 1 A-$ $\mathrm{Cr} 1 A-\mathrm{O} 3 A$ bite angle $82.34(7)^{\circ}$ is considerably less than $90^{\circ}$, while the folding angle of the cyclam in the $[\mathrm{Cr}(\mathrm{ox})(\text { cyclam })]^{+}$ cation is $98.97(8)^{\circ}$. The significant distortion of the octahedron and the larger folding angle in the $[\mathrm{Cr}(\mathrm{ox})(\text { cyclam })]^{+}$ cation seem to arise from the small bite angle of the bidentate oxalato ligand.

It is of interest to compare the conformation of the $\mathrm{Cr}_{2} \mathrm{O}_{7}{ }^{2-}$ anion with that found in other ionic crystals. In (I), the $\mathrm{Cr}_{2} \mathrm{O}_{7}{ }^{2-}$ anion exhibits a staggered conformation whereas a nearly eclipsed conformation is observed for $\left(\mathrm{C}_{9} \mathrm{H}_{14} \mathrm{~N}\right)_{2}\left[\mathrm{Cr}_{2} \mathrm{O}_{7}\right]$ and $\left(\mathrm{C}_{10} \mathrm{H}_{22} \mathrm{~N}_{2}\right)\left[\mathrm{Cr}_{2} \mathrm{O}_{7}\right]$, when viewed along the backbone of the dichromate anion (Trabelsi et al., 2015; Chebbi et al., 2016). This structural conformation of dichromate seems to depend on the size of the associated countercation (Moon et al., 2015, 2017). The $\mathrm{O} 1 B-\mathrm{Cr} 2 B-\mathrm{O}$ bond angles range from 107.1 (3) to $117.0(3)^{\circ}$; while the terminal $\mathrm{Cr} 2 B-\mathrm{O}$ bond lengths vary from 1.572 (12) to 1.673 (5) $\AA$, with a mean terminal $\mathrm{Cr} 2 B-\mathrm{O}$ bond length of $1.627 \AA$. The bridging $\mathrm{Cr} 2 B-\mathrm{O} 1 B$ bond is 1.684 (4) $\AA$ long, with the $\mathrm{Cr} 2 B-\mathrm{O} 1 B-\mathrm{Cr} 2 B(-x+2,-y,-z+1)$ bond angle of $136.0(3)^{\circ}$. These values are similar to those reported for the anions in the structures of $\left[\mathrm{Cr}(\mathrm{urea})_{6}\right]\left[\mathrm{Cr}_{2} \mathrm{O}_{7}\right] \mathrm{Br} \cdot \mathrm{H}_{2} \mathrm{O}$ (Moon et al., 2015) and $\left[\mathrm{Cr}(\mathrm{NCS})_{2} \text { (cyclam) }\right]_{2}\left[\mathrm{Cr}_{2} \mathrm{O}_{7}\right] \cdot \mathrm{H}_{2} \mathrm{O}$ (Moon et al., 2017). A further distortion of the anion undoubtedly results from its involvement in hydrogen-bonding interactions with the solvent water molecules (see Supramolecular features).

\section{Supramolecular features}

In the asymmetric unit, $\mathrm{O}-\mathrm{H} \cdots \mathrm{O}$ and $\mathrm{N}-\mathrm{H} \cdots \mathrm{O}$ hydrogen bonds link the water molecules to the $\mathrm{Cr}_{2} \mathrm{O}_{7}{ }^{2-}$ anion, 
Table 1

Hydrogen-bond geometry $\left(\AA,^{\circ}\right)$.

\begin{tabular}{lllll}
\hline$D-\mathrm{H} \cdots A$ & $D-\mathrm{H}$ & $\mathrm{H} \cdots A$ & $D \cdots A$ & $D-\mathrm{H} \cdots A$ \\
\hline $\mathrm{N} 1 A-\mathrm{H} 1 A \cdots \mathrm{O} 4 A^{\mathrm{i}}$ & 0.98 & 1.99 & $2.804(3)$ & 139 \\
$\mathrm{~N} 2 A-\mathrm{H} 2 A \cdots \mathrm{O} 1 S$ & 0.98 & 2.00 & $2.894(3)$ & 150 \\
$\mathrm{~N} 3 A-\mathrm{H} 3 A \cdots \mathrm{O} 2 A^{\mathrm{i}}$ & 0.98 & 1.89 & $2.842(3)$ & 163 \\
$\mathrm{~N} 4 A-\mathrm{H} 4 A \cdots \mathrm{O} 2 B 1^{\mathrm{ii}}$ & 0.98 & 2.25 & $3.100(16)$ & 144 \\
$\mathrm{~N} 4 A-\mathrm{H} 4 A \cdots \mathrm{O} 4 B 1^{\mathrm{ii}}$ & 0.98 & 2.37 & $3.108(6)$ & 132 \\
$\mathrm{~N} 4 A-\mathrm{H} 4 A \cdots \mathrm{O} 4 B 2^{\mathrm{ii}}$ & 0.98 & 2.15 & $3.05(2)$ & 151 \\
$\mathrm{O} 1 S-\mathrm{H} 1 O 1 \cdots \mathrm{O} 4 B 1$ & $0.85(1)$ & $2.33(5)$ & $2.876(6)$ & $123(5)$ \\
$\mathrm{O} 1 S-\mathrm{H} 1 O 1 \cdots \mathrm{O} 3 B 2$ & $0.85(1)$ & $2.20(4)$ & $2.903(10)$ & $141(5)$ \\
$\mathrm{O} 2 S-\mathrm{H} 2 O 2 \cdots \mathrm{O} 3 S$ & $0.85(1)$ & $1.91(2)$ & $2.729(6)$ & $164(6)$ \\
$\mathrm{O} 3 S-\mathrm{H} 1 O 3 \cdots \mathrm{O} 3 B 1$ & $0.85(1)$ & $1.93(3)$ & $2.725(6)$ & $156(7)$ \\
$\mathrm{O} 3 S-\mathrm{H} 1 O 3 \cdots \mathrm{O} 3 B 2$ & $0.85(1)$ & $2.28(2)$ & $3.113(10)$ & $167(6)$ \\
$\mathrm{O} 3 S-\mathrm{H} 2 O 3 \cdots \mathrm{O} 1 A^{\mathrm{iii}}$ & $0.85(1)$ & $2.52(3)$ & $3.301(4)$ & $154(6)$ \\
$\mathrm{O} 3 S-\mathrm{H} 2 O 3 \cdots \mathrm{O} 2 A^{\mathrm{iii}}$ & $0.85(1)$ & $2.21(5)$ & $2.911(5)$ & $140(6)$ \\
$\mathrm{O} 4 S-\mathrm{H} 1 O 4 \cdots \mathrm{O} 4 A$ & $0.84(1)$ & $2.11(4)$ & $2.834(4)$ & $144(6)$ \\
$\mathrm{O} 4 S-\mathrm{H} 2 O 4 \cdots \mathrm{O} 2 S$ & $0.85(1)$ & $1.89(2)$ & $2.723(5)$ & $166(6)$ \\
\hline & & & &
\end{tabular}

Symmetry codes: (i) $x-1, y, z$; (ii) $x,-y+\frac{1}{2}, z+\frac{1}{2}$; (iii) $x,-y+\frac{1}{2}, z-\frac{1}{2}$.

$[\mathrm{Cr}(\mathrm{ox})(\mathrm{cyclam})]^{+}$cation and other water molecules, while $\mathrm{N}-\mathrm{H} \cdots \mathrm{O}$ hydrogen bonds involving the cyclam $\mathrm{N}-\mathrm{H}$ groups and the $\mathrm{O}$ atoms of oxalate interconnect two $[\mathrm{Cr}(\mathrm{ox})(\mathrm{cy}-$ clam) $]^{+}$cations (Table 1, Figs. 1 and 2). An extensive array of these contacts generate a three-dimensional network of molecules (Fig. 2), and these hydrogen-bonding interactions help to stabilize the crystal structure.

\section{Database survey}

A search of the Cambridge Structural Database (Version 5.37, Feb 2016 with two updates; Groom et al., 2016) gave just one hit for a $\left[\mathrm{Cr}\left(\mathrm{C}_{2} \mathrm{O}_{4}\right)\left(\mathrm{C}_{10} \mathrm{H}_{24} \mathrm{~N}_{4}\right)\right]^{+}$unit, namely the complex $\left[\mathrm{Cr}(\right.$ ox $)($ cyclam $) \mathrm{ClO}_{4}$ (Choi et al., 2004b). However, the

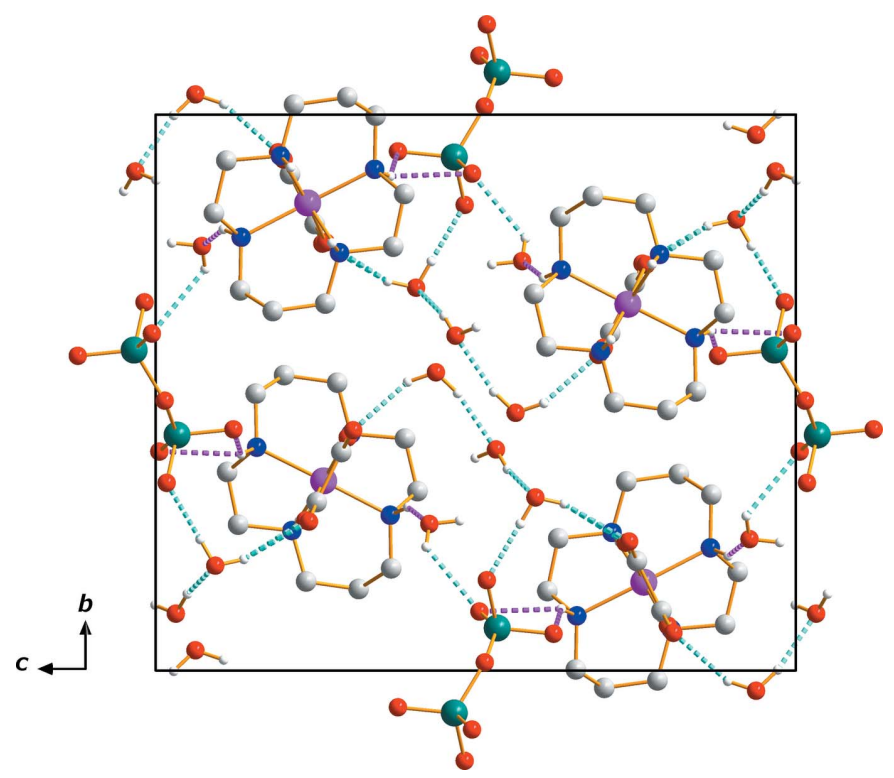

Figure 2

The crystal packing in compound (I), viewed perpendicular to the $b c$ plane. Dashed lines represent $\mathrm{N}-\mathrm{H} \cdots \mathrm{O}$ (pink) and $\mathrm{O}-\mathrm{H} \cdots \mathrm{O}$ (cyan) hydrogen-bonding interactions, respectively. $\mathrm{C}$-bound $\mathrm{H}$ atoms have been omitted.

Table 2

Experimental details.

Crystal data

Chemical formula

$M_{\mathrm{r}}$

Crystal system, space group

Temperature $(\mathrm{K})$

$a, b, c(\AA)$

$\beta\left(^{\circ}\right)$

$V\left(\AA^{3}\right)$

Z

Radiation type

$\mu\left(\mathrm{mm}^{-1}\right)$

Crystal size (mm)

Data collection

Diffractometer

Absorption correction

$T_{\min }, T_{\max }$

No. of measured, independent and observed $[I>2 \sigma(I)]$ reflections

$R_{\text {int }}$

$(\sin \theta / \lambda)_{\max }\left(\AA^{-1}\right)$

Refinement

$R\left[F^{2}>2 \sigma\left(F^{2}\right)\right], w R\left(F^{2}\right), S$

No. of reflections

No. of parameters

No. of restraints

$\mathrm{H}$-atom treatment

$\Delta \rho_{\max }, \Delta \rho_{\min }\left(\mathrm{e} \AA^{-3}\right)$

Computer programs: PAL BL2D-SMDC (Shin et al., 2016), HKL3000sm (Otwinowski \& Minor, 1997), SHELXT2014 (Sheldrick, 2015a), SHELXL2016 (Sheldrick, 2015b), DIAMOND 4 (Putz \& Brandenburg, 2014) and publCIF (Westrip, 2010).

structure of $\left[\mathrm{CrCl}_{2}\right.$ (cyclam) $)[\mathrm{Cr}(\mathrm{ox})($ cyclam $)]\left(\mathrm{ClO}_{4}\right)_{2}$ (Moon $\&$ Choi, 2016b) has also been reported recently. Until now, no structure of the $[\mathrm{Cr}(\mathrm{ox})(\text { cyclam })]^{+}$cation with a dichromate counter-anion has been deposited.

\section{Synthesis and crystallization}

The free ligand cyclam (98\%) was purchased from SigmaAldrich and used without further purification. All chemicals were reagent grade materials, and were used as received. The starting material, $[\mathrm{Cr}(\mathrm{ox})(\mathrm{cyclam})] \mathrm{ClO}_{4}$ was prepared according to the literature method (House \& McKee, 1984). The perchlorate salt of the complex $(0.03 \mathrm{~g})$ was dissolved in $10 \mathrm{~mL}$ of distilled water at $347 \mathrm{~K}$. The solution was filtered and the filtrate was added to $5 \mathrm{~mL}$ of water containing solid $\mathrm{K}_{2} \mathrm{Cr}_{2} \mathrm{O}_{7}$ (0.02 g). Orange block-like crystals of (I) suitable for $\mathrm{X}$-ray structural analysis were obtained after one week of slow evaporation at room temperature.

\section{Refinement}

Crystal data, data collection and structure refinement details are summarized in Table 2. All $\mathrm{H}$ atoms were placed in geometrically idealized positions and constrained to ride on 
their parent atoms, with $\mathrm{C}-\mathrm{H}=0.97 \AA$ and $\mathrm{N}-\mathrm{H}=0.98 \AA$, and with $U_{\text {iso }}(\mathrm{H})$ values of $1.2 U_{\text {eq }}$ of the parent atoms. The hydrogen atoms of the solvent water molecules were assigned based on a difference-Fourier map, and the $\mathrm{O}-\mathrm{H}$ distance and the $\mathrm{H}-\mathrm{O}-\mathrm{H}$ angle were restrained using DFIX and DANG constraints. The terminal $\mathrm{O}$ atoms of the dichromate anion are positionally disordered over two sets of sites. The occupancies of the respective pairs, $\mathrm{O} 2 B 1 / \mathrm{O} 2 B 2, \mathrm{O} 3 B 1 / \mathrm{O} 3 B 2$ and $\mathrm{O} 4 B 1 / \mathrm{O} 4 B 2$, were refined freely and, for the $\mathrm{O} 2 B 2$ and $\mathrm{O} 3 B 2$ atoms, ISOR restraints were applied. The occupancy ratio refined to 0.586 (6):0.414 (6). The bridging $\mathrm{O} 1 B$ atom of the dichromate anion is also disordered, in this case about the inversion centre. Consequently the components were refined at half-occupancy. The bridging atoms $\mathrm{O} 1 B / \mathrm{O} 1 B(-x+2,-y$, $-z+1)$ sites were refined using EXYZ/EADP restraints.

\section{Acknowledgements}

This work was supported by a grant from 2017 Research Funds of Andong National University. The X-ray crystallography experiment at PLS-II BL2D-SMC beamline was supported in part by MSIP and POSTECH.

\section{References}

Chebbi, H., Ben Smail, R. \& Zid, M. F. (2016). J. Struct. Chem. 57, 632-635.

Choi, J.-H. (2009). Inorg. Chim. Acta, 362, 4231-4236.

Choi, J.-H., Oh, I.-G., Lim, W.-T. \& Park, K.-M. (2004a). Acta Cryst. C60, m238-m240.

Choi, J.-H., Oh, I.-G., Suzuki, T. \& Kaizaki, S. (2004b). J. Mol. Struct. 694, 39-44.

De Clercq, E. (2010). J. Med. Chem. 53, 1438-1450.

De Leo, M. A., Bu, X., Bentow, J. \& Ford, P. C. (2000). Inorg. Chim. Acta, 300-302, 944-950.
Friesen, D. A., Quail, J. W., Waltz, W. L. \& Nashiem, R. E. (1997). Acta Cryst. C53, 687-691.

Groom, C. R., Bruno, I. J., Lightfoot, M. P. \& Ward, S. C. (2016). Acta Cryst. B72, 171-179.

Guzel, P., Aydın, Y. A. \& Deveci Aksoy, N. (2016). Int. J. Environ. Sci. Technol. 13, 1277-1288.

House, D. A. \& McKee, V. (1984). Inorg. Chem. 23, 4237-4242.

Meyer, K., Bendix, J., Bill, E., Weyhermüller, T. \& Wieghardt, K. (1998). Inorg. Chem. 37, 5180-5188.

Moon, D. \& Choi, J.-H. (2016a). Acta Cryst. E72, 456-459.

Moon, D. \& Choi, J.-H. (2016b). Acta Cryst. E72, 1417-1420.

Moon, D., Choi, J.-H., Ryoo, K. S. \& Hong, Y. P. (2013). Acta Cryst. E69, m376-m377.

Moon, D., Takase, M., Akitsu, T. \& Choi, J.-H. (2017). Acta Cryst. E73, 72-75.

Moon, D., Tanaka, S., Akitsu, T. \& Choi, J.-H. (2015). Acta Cryst. E71, 1336-1339.

Otwinowski, Z. \& Minor, W. (1997). Methods in Enzymology, Vol. 276, Macromolecular Crystallography, Part A, edited by C. W. Carter Jr \& R. M. Sweet, pp. 307-326. New York: Academic Press.

Poon, C. K. \& Pun, K. C. (1980). Inorg. Chem. 19, 568-569.

Putz, H. \& Brandenburg, K. (2014). DIAMOND. Crystal Impact GbR, Bonn, Germany.

Ronconi, L. \& Sadler, P. J. (2007). Coord. Chem. Rev. 251, 1633-1648.

Ross, A., Choi, J.-H., Hunter, T. M., Pannecouque, C., Moggach, S. A., Parsons, S., De Clercq, E. \& Sadler, P. J. (2012). Dalton Trans. 41, 6408-6418.

Sheldrick, G. M. (2015a). Acta Cryst. A71, 3-8.

Sheldrick, G. M. (2015b). Acta Cryst. C71, 3-8.

Shin, J. W., Eom, K. \& Moon, D. (2016). J. Synchrotron Rad. 23, 369373.

Subhan, M. A., Choi, J.-H. \& Ng, S. W. (2011). Z. Anorg. Allg. Chem. 637, 2193-2197.

Taylor, D. (1978). Aust. J. Chem. 31, 1455-1462.

Trabelsi, S., Roisnel, T. \& Marouani, H. (2015). J. Advan. Chem. 11, 3394-3403.

Westrip, S. P. (2010). J. Appl. Cryst. 43, 920-925.

Yusof, A. M. \& Malek, N. A. N. N. (2009). J. Hazard. Mater. 162, 1019-1024. 


\section{supporting information}

Acta Cryst. (2017). E73, 403-406 [https://doi.org/10.1107/S2056989017002614]

Crystal structure of bis[(oxalato- $\left.\kappa^{2} O^{1}, O^{2}\right)(1,4,8,11$-tetraazacyclotetradecane$\left.\kappa^{4} N\right)$ chromium(III)] dichromate octahydrate from synchrotron X-ray data

\section{Dohyun Moon and Jong-Ha Choi}

Computing details

Data collection: PAL BL2D-SMDC (Shin et al., 2016); cell refinement: HKL3000sm (Otwinowski \& Minor, 1997); data reduction: HKL3000sm (Otwinowski \& Minor, 1997); program(s) used to solve structure: SHELXT2014 (Sheldrick, 2015a); program(s) used to refine structure: SHELXL2016 (Sheldrick, 2015b); molecular graphics: DIAMOND 4 (Putz \& Brandenburg, 2014); software used to prepare material for publication: publCIF (Westrip, 2010).

$\operatorname{Bis}\left[\left(\right.\right.$ oxalato- $\left.\kappa^{2} O^{1}, O^{2}\right)\left(1,4,8,11\right.$-tetraazacyclotetradecane- $\left.\kappa^{4} N\right)$ chromium(III)] dichromate octahydrate

Crystal data

$\left[\mathrm{Cr}\left(\mathrm{C}_{2} \mathrm{O}_{4}\right)\left(\mathrm{C}_{10} \mathrm{H}_{24} \mathrm{~N}_{4}\right)\right]_{2}\left[\mathrm{Cr}_{2} \mathrm{O}_{7}\right] \cdot 8 \mathrm{H}_{2} \mathrm{O}$

$M_{r}=1040.83$

Monoclinic, $P 2_{1} / c$

$a=7.8270(16) \AA$

$b=15.407(3) \AA$

$c=18.086(4) \AA$

$\beta=100.86(3)^{\circ}$

$V=2141.9(8) \AA^{3}$

$Z=2$

Data collection

ADSC Q210 CCD area detector diffractometer

Radiation source: PLSII 2D bending magnet

$\omega$ scan

Absorption correction: empirical (using intensity measurements)

(HKL3000sm SCALEPACK; Otwinowski \& Minor, 1997)

$T_{\min }=0.889, T_{\max }=1.000$

Refinement

Refinement on $F^{2}$

Least-squares matrix: full

$R\left[F^{2}>2 \sigma\left(F^{2}\right)\right]=0.048$

$w R\left(F^{2}\right)=0.145$

$S=1.04$

5775 reflections

324 parameters

24 restraints

Hydrogen site location: mixed
$F(000)=1088$

$D_{\mathrm{x}}=1.614 \mathrm{Mg} \mathrm{m}^{-3}$

Synchrotron radiation, $\lambda=0.610 \AA$

Cell parameters from 63673 reflections

$\theta=0.4-33.7^{\circ}$

$\mu=0.71 \mathrm{~mm}^{-1}$

$T=298 \mathrm{~K}$

Block, orange

$0.15 \times 0.09 \times 0.08 \mathrm{~mm}$

21270 measured reflections

5775 independent reflections

4844 reflections with $I>2 \sigma(I)$

$R_{\text {int }}=0.027$

$\theta_{\max }=25.0^{\circ}, \theta_{\min }=2.3^{\circ}$

$h=-10 \rightarrow 10$

$k=-20 \rightarrow 20$

$l=-25 \rightarrow 25$

$\mathrm{H}$ atoms treated by a mixture of independent and constrained refinement

$w=1 /\left[\sigma^{2}\left(F_{\mathrm{o}}^{2}\right)+(0.0902 P)^{2}+1.1358 P\right]$ where $P=\left(F_{\mathrm{o}}{ }^{2}+2 F_{\mathrm{c}}{ }^{2}\right) / 3$

$(\Delta / \sigma)_{\max }=0.002$

$\Delta \rho_{\max }=1.62 \mathrm{e} \AA^{-3}$

$\Delta \rho_{\min }=-0.69$ e $\AA^{-3}$ 
Extinction correction: SHELXL2016 (Sheldrick, 2015b),

$\mathrm{Fc}^{*}=\mathrm{kFc}\left[1+0.001 \mathrm{xFc}^{2} \lambda^{3} / \sin (2 \theta)\right]^{-1 / 4}$

Extinction coefficient: 0.037 (3)

Special details

Geometry. All esds (except the esd in the dihedral angle between two 1.s. planes) are estimated using the full covariance matrix. The cell esds are taken into account individually in the estimation of esds in distances, angles and torsion angles; correlations between esds in cell parameters are only used when they are defined by crystal symmetry. An approximate (isotropic) treatment of cell esds is used for estimating esds involving l.s. planes.

Fractional atomic coordinates and isotropic or equivalent isotropic displacement parameters $\left(\AA^{2}\right)$

\begin{tabular}{|c|c|c|c|c|c|}
\hline & $x$ & $y$ & $z$ & $U_{\text {iso }} * / U_{\text {eq }}$ & Occ. $(<1)$ \\
\hline $\mathrm{Cr} 1 \mathrm{~A}$ & $0.38205(4)$ & $0.34112(2)$ & $0.73752(2)$ & $0.02641(13)$ & \\
\hline O1A & 0.59297 (19) & $0.27010(12)$ & $0.76591(10)$ & $0.0396(4)$ & \\
\hline $\mathrm{O} 2 \mathrm{~A}$ & $0.8739(2)$ & $0.26797(16)$ & $0.76043(14)$ & $0.0626(6)$ & \\
\hline $\mathrm{O} 3 \mathrm{~A}$ & $0.54391(19)$ & $0.41929(11)$ & $0.69995(10)$ & $0.0396(4)$ & \\
\hline $\mathrm{O} 4 \mathrm{~A}$ & $0.8222(2)$ & $0.43225(16)$ & $0.69140(14)$ & $0.0598(6)$ & \\
\hline N1A & $0.1816(2)$ & $0.42579(13)$ & $0.69489(12)$ & $0.0378(4)$ & \\
\hline H1A & 0.075547 & 0.405210 & 0.710638 & $0.045^{*}$ & \\
\hline $\mathrm{N} 2 \mathrm{~A}$ & $0.3113(2)$ & $0.27935(15)$ & $0.63360(12)$ & $0.0409(4)$ & \\
\hline $\mathrm{H} 2 \mathrm{~A}$ & 0.403674 & 0.293806 & 0.605848 & $0.049^{*}$ & \\
\hline N3A & $0.2422(2)$ & $0.25158(13)$ & $0.78721(12)$ & $0.0390(4)$ & \\
\hline $\mathrm{H} 3 \mathrm{~A}$ & 0.119637 & 0.269026 & 0.775430 & $0.047^{*}$ & \\
\hline N4A & $0.4158(3)$ & $0.40170(14)$ & $0.84214(11)$ & $0.0406(4)$ & \\
\hline $\mathrm{H} 4 \mathrm{~A}$ & 0.535961 & 0.389115 & 0.866550 & $0.049^{*}$ & \\
\hline $\mathrm{C} 1 \mathrm{~A}$ & $0.1568(4)$ & $0.4172(2)$ & $0.61046(18)$ & $0.0582(8)$ & \\
\hline H1AA & 0.049076 & 0.445014 & 0.586857 & $0.070^{*}$ & \\
\hline $\mathrm{H} 1 \mathrm{AB}$ & 0.252088 & 0.445169 & 0.592350 & $0.070^{*}$ & \\
\hline $\mathrm{C} 2 \mathrm{~A}$ & $0.1514(4)$ & $0.3226(2)$ & $0.59104(18)$ & $0.0612(8)$ & \\
\hline H2AA & 0.048592 & 0.296162 & 0.604142 & $0.073^{*}$ & \\
\hline $\mathrm{H} 2 \mathrm{AB}$ & 0.145782 & 0.315414 & 0.537358 & $0.073^{*}$ & \\
\hline $\mathrm{C} 3 \mathrm{~A}$ & $0.3008(4)$ & $0.1838(2)$ & $0.6321(2)$ & $0.0609(8)$ & \\
\hline H3AA & 0.417078 & 0.160207 & 0.647097 & $0.073^{*}$ & \\
\hline $\mathrm{H} 3 \mathrm{AB}$ & 0.256590 & 0.165122 & 0.580818 & $0.073^{*}$ & \\
\hline $\mathrm{C} 4 \mathrm{~A}$ & $0.1857(4)$ & $0.1466(2)$ & $0.6829(2)$ & $0.0638(9)$ & \\
\hline H4AA & 0.071236 & 0.172674 & 0.669584 & $0.077^{*}$ & \\
\hline $\mathrm{H} 4 \mathrm{AB}$ & 0.172624 & 0.084736 & 0.673435 & $0.077^{*}$ & \\
\hline $\mathrm{C} 5 \mathrm{~A}$ & $0.2513(4)$ & $0.16009(18)$ & $0.7649(2)$ & $0.0586(8)$ & \\
\hline H5AA & 0.183071 & 0.125105 & 0.793222 & $0.070^{*}$ & \\
\hline H5AB & 0.371013 & 0.140530 & 0.777515 & $0.070^{*}$ & \\
\hline C6A & $0.3031(4)$ & $0.2623(2)$ & $0.87113(18)$ & $0.0597(8)$ & \\
\hline H6AA & 0.224823 & 0.232351 & 0.898189 & $0.072 *$ & \\
\hline H6AB & 0.418710 & 0.238033 & 0.886411 & $0.072 *$ & \\
\hline C7A & $0.3053(5)$ & $0.3571(3)$ & $0.88827(18)$ & $0.0640(8)$ & \\
\hline H7AA & 0.187953 & 0.380198 & 0.876854 & $0.077^{*}$ & \\
\hline $\mathrm{H} 7 \mathrm{AB}$ & 0.351179 & 0.366653 & 0.941312 & $0.077^{*}$ & \\
\hline $\mathrm{C} 8 \mathrm{~A}$ & $0.3993(4)$ & $0.4980(2)$ & $0.8437(2)$ & $0.0605(8)$ & \\
\hline
\end{tabular}




\begin{tabular}{|c|c|c|c|c|c|}
\hline H8AA & 0.493986 & 0.523712 & 0.823521 & $0.073 *$ & \\
\hline H8AB & 0.410781 & 0.516864 & 0.895598 & $0.073^{*}$ & \\
\hline $\mathrm{C} 9 \mathrm{~A}$ & $0.2285(4)$ & $0.5313(2)$ & $0.7993(2)$ & $0.0657(9)$ & \\
\hline H9AA & 0.133959 & 0.502373 & 0.817229 & $0.079^{*}$ & \\
\hline H9AB & 0.219591 & 0.592861 & 0.809118 & $0.079 *$ & \\
\hline C10A & $0.2063(4)$ & $0.51784(19)$ & $0.7163(2)$ & $0.0595(8)$ & \\
\hline H10A & 0.106358 & 0.550800 & 0.691276 & $0.071^{*}$ & \\
\hline H10B & 0.308132 & 0.539863 & 0.699087 & $0.071^{*}$ & \\
\hline C11A & $0.7305(3)$ & $0.30305(18)$ & $0.74841(14)$ & $0.0415(5)$ & \\
\hline C12A & $0.7022(3)$ & $0.39281(18)$ & $0.70995(14)$ & $0.0412(5)$ & \\
\hline $\mathrm{Cr} 2 \mathrm{~B}$ & $0.86158(6)$ & $0.07641(3)$ & $0.46646(2)$ & $0.04561(15)$ & \\
\hline O1B & $0.9327(6)$ & -0.0145 & $0.5141(3)$ & $0.0780(16)$ & 0.5 \\
\hline $\mathrm{O} 2 \mathrm{~B} 1$ & $0.814(2)$ & $0.0661(9)$ & $0.3785(7)$ & $0.068(3)$ & $0.586(6)$ \\
\hline O3B1 & $0.9854(7)$ & $0.1624(3)$ & $0.4827(3)$ & $0.0797(17)$ & $0.586(6)$ \\
\hline O4B1 & $0.6779(6)$ & $0.1061(4)$ & $0.4942(3)$ & $0.0811(16)$ & $0.586(6)$ \\
\hline $\mathrm{O} 2 \mathrm{~B} 2$ & $0.7152(9)$ & $0.0097(6)$ & $0.4864(4)$ & $0.093(3)$ & $0.414(6)$ \\
\hline O3B2 & 0.8779 (13) & $0.1556(6)$ & $0.5165(5)$ & $0.097(3)$ & $0.414(6)$ \\
\hline O4B2 & $0.812(3)$ & $0.0895(14)$ & $0.3789(11)$ & $0.075(5)$ & $0.414(6)$ \\
\hline O1S & $0.6259(5)$ & $0.2636(2)$ & $0.57243(19)$ & $0.0909(10)$ & \\
\hline $\mathrm{H} 1 \mathrm{O} 1$ & $0.698(7)$ & $0.222(3)$ & $0.577(3)$ & $0.136^{*}$ & \\
\hline $\mathrm{H} 2 \mathrm{O} 1$ & $0.596(8)$ & $0.269(4)$ & $0.5252(8)$ & $0.136^{*}$ & \\
\hline $\mathrm{O} 2 \mathrm{~S}$ & $0.6340(5)$ & $0.3977(2)$ & $0.4684(2)$ & $0.0935(9)$ & \\
\hline $\mathrm{H} 1 \mathrm{O} 2$ & $0.576(7)$ & $0.382(4)$ & $0.501(3)$ & $0.140^{*}$ & \\
\hline $\mathrm{H} 2 \mathrm{O} 2$ & $0.698(7)$ & $0.362(3)$ & $0.450(3)$ & $0.140^{*}$ & \\
\hline $\mathrm{O} 3 \mathrm{~S}$ & $0.8777(6)$ & $0.3113(2)$ & $0.4068(2)$ & $0.1014(11)$ & \\
\hline $\mathrm{H} 1 \mathrm{O} 3$ & $0.880(9)$ & $0.264(2)$ & $0.431(3)$ & $0.152 *$ & \\
\hline $\mathrm{H} 2 \mathrm{O} 3$ & $0.827(8)$ & $0.300(4)$ & $0.3622(14)$ & $0.152 *$ & \\
\hline $\mathrm{O} 4 \mathrm{~S}$ & $0.7101(5)$ & $0.5364(2)$ & $0.56219(18)$ & $0.0920(9)$ & \\
\hline $\mathrm{H} 1 \mathrm{O} 4$ & $0.708(9)$ & $0.519(3)$ & $0.6063(12)$ & $0.138^{*}$ & \\
\hline $\mathrm{H} 2 \mathrm{O} 4$ & $0.675(8)$ & $0.499(3)$ & $0.528(2)$ & $0.138^{*}$ & \\
\hline
\end{tabular}

Atomic displacement parameters $\left(\AA^{2}\right)$

\begin{tabular}{lllllll}
\hline & $U^{11}$ & $U^{22}$ & $U^{33}$ & $U^{12}$ & $U^{13}$ & $U^{23}$ \\
\hline Cr1A & $0.01035(16)$ & $0.0353(2)$ & $0.03406(19)$ & $0.00068(10)$ & $0.00548(11)$ & $-0.00334(12)$ \\
O1A & $0.0188(7)$ & $0.0472(10)$ & $0.0521(9)$ & $0.0076(6)$ & $0.0050(6)$ & $-0.0005(7)$ \\
O2A & $0.0197(8)$ & $0.0833(16)$ & $0.0835(15)$ & $0.0165(8)$ & $0.0066(8)$ & $-0.0129(12)$ \\
O3A & $0.0177(7)$ & $0.0490(10)$ & $0.0546(10)$ & $-0.0027(6)$ & $0.0133(6)$ & $0.0023(7)$ \\
O4A & $0.0229(8)$ & $0.0871(16)$ & $0.0737(14)$ & $-0.0124(9)$ & $0.0203(8)$ & $-0.0010(11)$ \\
N1A & $0.0173(8)$ & $0.0424(11)$ & $0.0544(11)$ & $0.0062(7)$ & $0.0089(7)$ & $0.0063(8)$ \\
N2A & $0.0258(9)$ & $0.0561(12)$ & $0.0409(10)$ & $-0.0004(8)$ & $0.0066(7)$ & $-0.0124(9)$ \\
N3A & $0.0216(8)$ & $0.0435(11)$ & $0.0537(11)$ & $-0.0007(7)$ & $0.0115(8)$ & $0.0059(8)$ \\
N4A & $0.0273(9)$ & $0.0533(12)$ & $0.0408(10)$ & $-0.0009(8)$ & $0.0058(7)$ & $-0.0127(8)$ \\
C1A & $0.0373(13)$ & $0.080(2)$ & $0.0565(16)$ & $0.0114(13)$ & $0.0069(11)$ & $0.0225(15)$ \\
C2A & $0.0434(15)$ & $0.087(2)$ & $0.0473(15)$ & $0.0015(15)$ & $-0.0069(12)$ & $-0.0098(14)$ \\
C3A & $0.0495(16)$ & $0.0600(18)$ & $0.073(2)$ & $-0.0032(13)$ & $0.0114(14)$ & $-0.0286(15)$ \\
C4A & $0.0488(17)$ & $0.0501(17)$ & $0.093(2)$ & $-0.0108(12)$ & $0.0130(16)$ & $-0.0183(15)$ \\
C5A & $0.0442(15)$ & $0.0407(15)$ & $0.092(2)$ & $-0.0025(10)$ & $0.0169(15)$ & $0.0052(13)$
\end{tabular}




\begin{tabular}{lllllll} 
C6A & $0.0497(16)$ & $0.077(2)$ & $0.0546(16)$ & $0.0013(14)$ & $0.0162(13)$ & $0.0168(14)$ \\
C7A & $0.067(2)$ & $0.086(2)$ & $0.0446(15)$ & $-0.0042(17)$ & $0.0243(14)$ & $-0.0113(14)$ \\
C8A & $0.0517(17)$ & $0.0561(18)$ & $0.074(2)$ & $-0.0037(13)$ & $0.0125(14)$ & $-0.0275(15)$ \\
C9A & $0.0498(17)$ & $0.0528(18)$ & $0.097(3)$ & $0.0099(13)$ & $0.0195(16)$ & $-0.0179(16)$ \\
C10A & $0.0376(14)$ & $0.0447(15)$ & $0.098(2)$ & $0.0084(11)$ & $0.0189(14)$ & $0.0074(14)$ \\
C11A & $0.0160(9)$ & $0.0608(15)$ & $0.0470(12)$ & $0.0055(9)$ & $0.0041(8)$ & $-0.0140(10)$ \\
C12A & $0.0177(9)$ & $0.0612(15)$ & $0.0472(12)$ & $-0.0071(9)$ & $0.0120(8)$ & $-0.0119(10)$ \\
Cr2B & $0.0406(2)$ & $0.0553(3)$ & $0.0379(2)$ & $0.00143(17)$ & $-0.00027(16)$ & $0.00325(16)$ \\
O1B & $0.058(3)$ & $0.083(3)$ & $0.094(4)$ & $0.032(3)$ & $0.016(3)$ & $0.051(3)$ \\
O2B1 & $0.074(5)$ & $0.082(6)$ & $0.042(4)$ & $0.010(4)$ & $-0.002(3)$ & $-0.009(4)$ \\
O3B1 & $0.076(3)$ & $0.065(3)$ & $0.086(3)$ & $-0.025(2)$ & $-0.016(3)$ & $-0.006(2)$ \\
O4B1 & $0.066(3)$ & $0.095(4)$ & $0.087(3)$ & $0.011(2)$ & $0.027(2)$ & $-0.005(3)$ \\
O2B2 & $0.065(4)$ & $0.126(6)$ & $0.094(5)$ & $-0.007(4)$ & $0.027(3)$ & $0.045(4)$ \\
O3B2 & $0.096(3)$ & $0.095(3)$ & $0.097(3)$ & $0.0059(19)$ & $0.0135(19)$ & $-0.0124(18)$ \\
O4B2 & $0.050(5)$ & $0.113(13)$ & $0.060(7)$ & $0.023(7)$ & $0.009(5)$ & $0.047(7)$ \\
O1S & $0.097(2)$ & $0.102(2)$ & $0.089(2)$ & $0.0078(17)$ & $0.0558(19)$ & $-0.0074(17)$ \\
O2S & $0.110(3)$ & $0.082(2)$ & $0.089(2)$ & $0.0060(18)$ & $0.0202(18)$ & $-0.0030(16)$ \\
O3S & $0.131(3)$ & $0.084(2)$ & $0.082(2)$ & $0.013(2)$ & $0.003(2)$ & $-0.0045(16)$ \\
O4S & $0.111(3)$ & $0.091(2)$ & $0.0739(18)$ & $0.0025(19)$ & $0.0187(19)$ & $-0.0037(15)$ \\
& & & & & & \\
\hline
\end{tabular}

Geometric parameters $\left(\AA,{ }^{\circ}\right)$

\begin{tabular}{llll}
\hline Cr1A-O3A & $1.9600(16)$ & $\mathrm{C} 5 \mathrm{~A}-\mathrm{H} 5 \mathrm{AB}$ & 0.9700 \\
$\mathrm{Cr} 1 \mathrm{~A}-\mathrm{O} 1 \mathrm{~A}$ & $1.9665(16)$ & $\mathrm{C} 6 \mathrm{~A}-\mathrm{C} 7 \mathrm{~A}$ & $1.493(5)$ \\
$\mathrm{Cr} 1 \mathrm{~A}-\mathrm{N} 3 \mathrm{~A}$ & $2.069(2)$ & $\mathrm{C} 6 \mathrm{~A}-\mathrm{H} 6 \mathrm{AA}$ & 0.9700 \\
$\mathrm{Cr} 1 \mathrm{~A}-\mathrm{N} 1 \mathrm{~A}$ & $2.0739(19)$ & $\mathrm{C} 6 \mathrm{~A}-\mathrm{H} 6 \mathrm{AB}$ & 0.9700 \\
$\mathrm{Cr} 1 \mathrm{~A}-\mathrm{N} 4 \mathrm{~A}$ & $2.081(2)$ & $\mathrm{C} 7 \mathrm{~A}-\mathrm{H} 7 \mathrm{AA}$ & 0.9700 \\
$\mathrm{Cr} 1 \mathrm{~A}-\mathrm{N} 2 \mathrm{~A}$ & $2.086(2)$ & $\mathrm{C} 7 \mathrm{~A}-\mathrm{H} 7 \mathrm{AB}$ & 0.9700 \\
$\mathrm{O} 1 \mathrm{~A}-\mathrm{C} 11 \mathrm{~A}$ & $1.283(3)$ & $\mathrm{C} 8 \mathrm{~A}-\mathrm{C} 9 \mathrm{~A}$ & $1.513(5)$ \\
$\mathrm{O} 2 \mathrm{~A}-\mathrm{C} 11 \mathrm{~A}$ & $1.228(3)$ & $\mathrm{C} 8 \mathrm{~A}-\mathrm{H} 8 \mathrm{AA}$ & 0.9700 \\
$\mathrm{O} 3 \mathrm{~A}-\mathrm{C} 12 \mathrm{~A}$ & $1.284(3)$ & $\mathrm{C} 8 \mathrm{~A}-\mathrm{H} 8 \mathrm{AB}$ & 0.9700 \\
$\mathrm{O} 4 \mathrm{~A}-\mathrm{C} 12 \mathrm{~A}$ & $1.218(3)$ & $\mathrm{C} 9 \mathrm{~A}-\mathrm{C} 10 \mathrm{~A}$ & $1.493(5)$ \\
$\mathrm{N} 1 \mathrm{~A}-\mathrm{C} 10 \mathrm{~A}$ & $1.473(4)$ & $\mathrm{C} 9 \mathrm{~A}-\mathrm{H} 9 \mathrm{AA}$ & 0.9700 \\
$\mathrm{~N} 1 \mathrm{~A}-\mathrm{C} 1 \mathrm{~A}$ & $1.508(4)$ & $\mathrm{C} 9 \mathrm{~A}-\mathrm{H} 9 \mathrm{AB}$ & 0.9700 \\
$\mathrm{~N} 1 \mathrm{~A}-\mathrm{H} 1 \mathrm{~A}$ & 0.9800 & $\mathrm{C} 10 \mathrm{~A}-\mathrm{H} 10 \mathrm{~A}$ & 0.9700 \\
$\mathrm{~N} 2 \mathrm{~A}-\mathrm{C} 3 \mathrm{~A}$ & $1.475(4)$ & $\mathrm{C} 10 \mathrm{~A}-\mathrm{H} 10 \mathrm{~B}$ & 0.9700 \\
$\mathrm{~N} 2 \mathrm{~A}-\mathrm{C} 2 \mathrm{~A}$ & $1.497(4)$ & $\mathrm{C} 11 \mathrm{~A}-\mathrm{C} 12 \mathrm{~A}$ & $1.545(4)$ \\
$\mathrm{N} 2 \mathrm{~A}-\mathrm{H} 2 \mathrm{~A}$ & 0.9800 & $\mathrm{Cr} 2 \mathrm{~B}-\mathrm{O} 3 \mathrm{~B} 2$ & $1.511(8)$ \\
$\mathrm{N} 3 \mathrm{~A}-\mathrm{C} 5 \mathrm{~A}$ & $1.472(4)$ & $\mathrm{Cr} 2 \mathrm{~B}-\mathrm{O} 4 \mathrm{~B} 2$ & $1.571(19)$ \\
$\mathrm{N} 3 \mathrm{~A}-\mathrm{C} 6 \mathrm{~A}$ & $1.512(4)$ & $\mathrm{Cr} 2 \mathrm{~B}-\mathrm{O} 2 \mathrm{~B} 1$ & $1.572(12)$ \\
$\mathrm{N} 3 \mathrm{~A}-\mathrm{H} 3 \mathrm{~A}$ & 0.9800 & $\mathrm{Cr} 2 \mathrm{~B}-\mathrm{O} 2 \mathrm{~B} 2$ & $1.630(7)$ \\
$\mathrm{N} 4 \mathrm{~A}-\mathrm{C} 7 \mathrm{~A}$ & $1.479(4)$ & $\mathrm{Cr} 2 \mathrm{~B}-\mathrm{O} 3 \mathrm{~B} 1$ & $1.635(4)$ \\
$\mathrm{N} 4 \mathrm{~A}-\mathrm{C} 8 \mathrm{~A}$ & $1.491(4)$ & $\mathrm{Cr} 2 \mathrm{~B}-\mathrm{O} 4 \mathrm{~B} 1$ & $1.673(5)$ \\
$\mathrm{N} 4 \mathrm{~A}-\mathrm{H} 4 \mathrm{~A}$ & 0.9800 & $\mathrm{Cr} 2 \mathrm{~B}-\mathrm{O} 1 \mathrm{~B}$ & $1.684(4)$ \\
$\mathrm{C} 1 \mathrm{~A}-\mathrm{C} 2 \mathrm{~A}$ & $1.498(5)$ & $\mathrm{Cr} 2 \mathrm{~B}-\mathrm{O} 1 \mathrm{~B}^{\mathrm{i}}$ & $1.847(4)$ \\
$\mathrm{C} 1 \mathrm{~A}-\mathrm{H} 1 \mathrm{AA}$ & 0.9700 & $\mathrm{O} 1 \mathrm{~B}-\mathrm{O} 1 \mathrm{~B}^{\mathrm{i}}$ & $1.332(10)$ \\
$\mathrm{C} 1 \mathrm{~A}-\mathrm{H} 1 \mathrm{AB}$ & 0.9700 & $\mathrm{O} 1 \mathrm{~B}-\mathrm{O} 2 \mathrm{~B} 2$ & $1.723(9)$ \\
$\mathrm{C} 2 \mathrm{~A}-\mathrm{H} 2 \mathrm{AA}$ & 0.9700 & $\mathrm{O} 1 \mathrm{~S}-\mathrm{H} 1 \mathrm{O} 1$ & $0.845(10)$ \\
& & &
\end{tabular}




\begin{tabular}{|c|c|c|c|}
\hline $\mathrm{C} 2 \mathrm{~A}-\mathrm{H} 2 \mathrm{AB}$ & 0.9700 & $\mathrm{O} 1 \mathrm{~S}-\mathrm{H} 2 \mathrm{O} 1$ & $0.847(10)$ \\
\hline $\mathrm{C} 3 \mathrm{~A}-\mathrm{C} 4 \mathrm{~A}$ & $1.516(5)$ & $\mathrm{O} 2 \mathrm{~S}-\mathrm{H} 1 \mathrm{O} 2$ & $0.841(10)$ \\
\hline $\mathrm{C} 3 \mathrm{~A}-\mathrm{H} 3 \mathrm{AA}$ & 0.9700 & $\mathrm{O} 2 \mathrm{~S}-\mathrm{H} 2 \mathrm{O} 2$ & $0.845(10)$ \\
\hline $\mathrm{C} 3 \mathrm{~A}-\mathrm{H} 3 \mathrm{AB}$ & 0.9700 & $\mathrm{O} 3 \mathrm{~S}-\mathrm{H} 1 \mathrm{O} 3$ & $0.845(10)$ \\
\hline $\mathrm{C} 4 \mathrm{~A}-\mathrm{C} 5 \mathrm{~A}$ & $1.489(5)$ & $\mathrm{O} 3 \mathrm{~S}-\mathrm{H} 2 \mathrm{O} 3$ & $0.848(10)$ \\
\hline $\mathrm{C} 4 \mathrm{~A}-\mathrm{H} 4 \mathrm{AA}$ & 0.9700 & $\mathrm{O} 4 \mathrm{~S}-\mathrm{H} 1 \mathrm{O} 4$ & $0.843(10)$ \\
\hline $\mathrm{C} 4 \mathrm{~A}-\mathrm{H} 4 \mathrm{AB}$ & 0.9700 & $\mathrm{O} 4 \mathrm{~S}-\mathrm{H} 2 \mathrm{O} 4$ & $0.851(10)$ \\
\hline $\mathrm{C} 5 \mathrm{~A}-\mathrm{H} 5 \mathrm{AA}$ & 0.9700 & & \\
\hline $\mathrm{O} 3 \mathrm{~A}-\mathrm{Cr} 1 \mathrm{~A}-\mathrm{O} 1 \mathrm{~A}$ & $82.34(7)$ & $\mathrm{N} 3 \mathrm{~A}-\mathrm{C} 5 \mathrm{~A}-\mathrm{H} 5 \mathrm{AB}$ & 109.1 \\
\hline $\mathrm{O} 3 \mathrm{~A}-\mathrm{Cr} 1 \mathrm{~A}-\mathrm{N} 3 \mathrm{~A}$ & $171.83(8)$ & $\mathrm{C} 4 \mathrm{~A}-\mathrm{C} 5 \mathrm{~A}-\mathrm{H} 5 \mathrm{AB}$ & 109.1 \\
\hline $\mathrm{O} 1 \mathrm{~A}-\mathrm{Cr} 1 \mathrm{~A}-\mathrm{N} 3 \mathrm{~A}$ & $90.14(8)$ & $\mathrm{H} 5 \mathrm{AA}-\mathrm{C} 5 \mathrm{~A}-\mathrm{H} 5 \mathrm{AB}$ & 107.8 \\
\hline $\mathrm{O} 3 \mathrm{~A}-\mathrm{Cr} 1 \mathrm{~A}-\mathrm{N} 1 \mathrm{~A}$ & $88.72(7)$ & $\mathrm{C} 7 \mathrm{~A}-\mathrm{C} 6 \mathrm{~A}-\mathrm{N} 3 \mathrm{~A}$ & $107.7(2)$ \\
\hline $\mathrm{O} 1 \mathrm{~A}-\mathrm{Cr} 1 \mathrm{~A}-\mathrm{N} 1 \mathrm{~A}$ & $170.42(8)$ & $\mathrm{C} 7 \mathrm{~A}-\mathrm{C} 6 \mathrm{~A}-\mathrm{H} 6 \mathrm{AA}$ & 110.2 \\
\hline $\mathrm{N} 3 \mathrm{~A}-\mathrm{Cr} 1 \mathrm{~A}-\mathrm{N} 1 \mathrm{~A}$ & $98.97(8)$ & $\mathrm{N} 3 \mathrm{~A}-\mathrm{C} 6 \mathrm{~A}-\mathrm{H} 6 \mathrm{AA}$ & 110.2 \\
\hline $\mathrm{O} 3 \mathrm{~A}-\mathrm{Cr} 1 \mathrm{~A}-\mathrm{N} 4 \mathrm{~A}$ & $93.44(8)$ & $\mathrm{C} 7 \mathrm{~A}-\mathrm{C} 6 \mathrm{~A}-\mathrm{H} 6 \mathrm{AB}$ & 110.2 \\
\hline $\mathrm{O} 1 \mathrm{~A}-\mathrm{Cr} 1 \mathrm{~A}-\mathrm{N} 4 \mathrm{~A}$ & $93.19(8)$ & $\mathrm{N} 3 \mathrm{~A}-\mathrm{C} 6 \mathrm{~A}-\mathrm{H} 6 \mathrm{AB}$ & 110.2 \\
\hline $\mathrm{N} 3 \mathrm{~A}-\mathrm{Cr} 1 \mathrm{~A}-\mathrm{N} 4 \mathrm{~A}$ & $83.74(9)$ & $\mathrm{H} 6 \mathrm{AA}-\mathrm{C} 6 \mathrm{~A}-\mathrm{H} 6 \mathrm{AB}$ & 108.5 \\
\hline $\mathrm{N} 1 \mathrm{~A}-\mathrm{Cr} 1 \mathrm{~A}-\mathrm{N} 4 \mathrm{~A}$ & $90.77(9)$ & $\mathrm{N} 4 \mathrm{~A}-\mathrm{C} 7 \mathrm{~A}-\mathrm{C} 6 \mathrm{~A}$ & $108.8(2)$ \\
\hline $\mathrm{O} 3 \mathrm{~A}-\mathrm{Cr} 1 \mathrm{~A}-\mathrm{N} 2 \mathrm{~A}$ & $92.69(8)$ & $\mathrm{N} 4 \mathrm{~A}-\mathrm{C} 7 \mathrm{~A}-\mathrm{H} 7 \mathrm{AA}$ & 109.9 \\
\hline $\mathrm{O} 1 \mathrm{~A}-\mathrm{Cr} 1 \mathrm{~A}-\mathrm{N} 2 \mathrm{~A}$ & $92.78(8)$ & $\mathrm{C} 6 \mathrm{~A}-\mathrm{C} 7 \mathrm{~A}-\mathrm{H} 7 \mathrm{AA}$ & 109.9 \\
\hline $\mathrm{N} 3 \mathrm{~A}-\mathrm{Cr} 1 \mathrm{~A}-\mathrm{N} 2 \mathrm{~A}$ & $90.86(9)$ & $\mathrm{N} 4 \mathrm{~A}-\mathrm{C} 7 \mathrm{~A}-\mathrm{H} 7 \mathrm{AB}$ & 109.9 \\
\hline $\mathrm{N} 1 \mathrm{~A}-\mathrm{Cr} 1 \mathrm{~A}-\mathrm{N} 2 \mathrm{~A}$ & $84.17(9)$ & $\mathrm{C} 6 \mathrm{~A}-\mathrm{C} 7 \mathrm{~A}-\mathrm{H} 7 \mathrm{AB}$ & 109.9 \\
\hline $\mathrm{N} 4 \mathrm{~A}-\mathrm{Cr} 1 \mathrm{~A}-\mathrm{N} 2 \mathrm{~A}$ & $171.96(8)$ & H7AA-C7A-H7AB & 108.3 \\
\hline $\mathrm{C} 11 \mathrm{~A}-\mathrm{O} 1 \mathrm{~A}-\mathrm{Cr} 1 \mathrm{~A}$ & $114.69(16)$ & $\mathrm{N} 4 \mathrm{~A}-\mathrm{C} 8 \mathrm{~A}-\mathrm{C} 9 \mathrm{~A}$ & $113.4(2)$ \\
\hline $\mathrm{C} 12 \mathrm{~A}-\mathrm{O} 3 \mathrm{~A}-\mathrm{Cr} 1 \mathrm{~A}$ & $115.17(17)$ & $\mathrm{N} 4 \mathrm{~A}-\mathrm{C} 8 \mathrm{~A}-\mathrm{H} 8 \mathrm{AA}$ & 108.9 \\
\hline $\mathrm{C} 10 \mathrm{~A}-\mathrm{N} 1 \mathrm{~A}-\mathrm{C} 1 \mathrm{~A}$ & $109.6(2)$ & $\mathrm{C} 9 \mathrm{~A}-\mathrm{C} 8 \mathrm{~A}-\mathrm{H} 8 \mathrm{AA}$ & 108.9 \\
\hline $\mathrm{C} 10 \mathrm{~A}-\mathrm{N} 1 \mathrm{~A}-\mathrm{Cr} 1 \mathrm{~A}$ & $117.10(17)$ & $\mathrm{N} 4 \mathrm{~A}-\mathrm{C} 8 \mathrm{~A}-\mathrm{H} 8 \mathrm{AB}$ & 108.9 \\
\hline $\mathrm{C} 1 \mathrm{~A}-\mathrm{N} 1 \mathrm{~A}-\mathrm{Cr} 1 \mathrm{~A}$ & $105.39(15)$ & $\mathrm{C} 9 \mathrm{~A}-\mathrm{C} 8 \mathrm{~A}-\mathrm{H} 8 \mathrm{AB}$ & 108.9 \\
\hline $\mathrm{C} 10 \mathrm{~A}-\mathrm{N} 1 \mathrm{~A}-\mathrm{H} 1 \mathrm{~A}$ & 108.1 & $\mathrm{H} 8 \mathrm{AA}-\mathrm{C} 8 \mathrm{~A}-\mathrm{H} 8 \mathrm{AB}$ & 107.7 \\
\hline $\mathrm{C} 1 \mathrm{~A}-\mathrm{N} 1 \mathrm{~A}-\mathrm{H} 1 \mathrm{~A}$ & 108.1 & $\mathrm{C} 10 \mathrm{~A}-\mathrm{C} 9 \mathrm{~A}-\mathrm{C} 8 \mathrm{~A}$ & $114.2(3)$ \\
\hline $\mathrm{Cr} 1 \mathrm{~A}-\mathrm{N} 1 \mathrm{~A}-\mathrm{H} 1 \mathrm{~A}$ & 108.1 & C10A-C9A-H9AA & 108.7 \\
\hline $\mathrm{C} 3 \mathrm{~A}-\mathrm{N} 2 \mathrm{~A}-\mathrm{C} 2 \mathrm{~A}$ & $113.4(2)$ & $\mathrm{C} 8 \mathrm{~A}-\mathrm{C} 9 \mathrm{~A}-\mathrm{H} 9 \mathrm{AA}$ & 108.7 \\
\hline $\mathrm{C} 3 \mathrm{~A}-\mathrm{N} 2 \mathrm{~A}-\mathrm{Cr} 1 \mathrm{~A}$ & $118.47(19)$ & $\mathrm{C} 10 \mathrm{~A}-\mathrm{C} 9 \mathrm{~A}-\mathrm{H} 9 \mathrm{AB}$ & 108.7 \\
\hline $\mathrm{C} 2 \mathrm{~A}-\mathrm{N} 2 \mathrm{~A}-\mathrm{Cr} 1 \mathrm{~A}$ & $108.32(17)$ & $\mathrm{C} 8 \mathrm{~A}-\mathrm{C} 9 \mathrm{~A}-\mathrm{H} 9 \mathrm{AB}$ & 108.7 \\
\hline $\mathrm{C} 3 \mathrm{~A}-\mathrm{N} 2 \mathrm{~A}-\mathrm{H} 2 \mathrm{~A}$ & 105.2 & H9AA-C9A-H9AB & 107.6 \\
\hline $\mathrm{C} 2 \mathrm{~A}-\mathrm{N} 2 \mathrm{~A}-\mathrm{H} 2 \mathrm{~A}$ & 105.2 & $\mathrm{~N} 1 \mathrm{~A}-\mathrm{C} 10 \mathrm{~A}-\mathrm{C} 9 \mathrm{~A}$ & $112.5(3)$ \\
\hline $\mathrm{Cr} 1 \mathrm{~A}-\mathrm{N} 2 \mathrm{~A}-\mathrm{H} 2 \mathrm{~A}$ & 105.2 & $\mathrm{~N} 1 \mathrm{~A}-\mathrm{C} 10 \mathrm{~A}-\mathrm{H} 10 \mathrm{~A}$ & 109.1 \\
\hline $\mathrm{C} 5 \mathrm{~A}-\mathrm{N} 3 \mathrm{~A}-\mathrm{C} 6 \mathrm{~A}$ & $111.0(2)$ & $\mathrm{C} 9 \mathrm{~A}-\mathrm{C} 10 \mathrm{~A}-\mathrm{H} 10 \mathrm{~A}$ & 109.1 \\
\hline $\mathrm{C} 5 \mathrm{~A}-\mathrm{N} 3 \mathrm{~A}-\mathrm{Cr} 1 \mathrm{~A}$ & $117.56(18)$ & $\mathrm{N} 1 \mathrm{~A}-\mathrm{C} 10 \mathrm{~A}-\mathrm{H} 10 \mathrm{~B}$ & 109.1 \\
\hline $\mathrm{C} 6 \mathrm{~A}-\mathrm{N} 3 \mathrm{~A}-\mathrm{Cr} 1 \mathrm{~A}$ & $105.70(17)$ & C9A-C10A-H10B & 109.1 \\
\hline $\mathrm{C} 5 \mathrm{~A}-\mathrm{N} 3 \mathrm{~A}-\mathrm{H} 3 \mathrm{~A}$ & 107.4 & $\mathrm{H} 10 \mathrm{~A}-\mathrm{C} 10 \mathrm{~A}-\mathrm{H} 10 \mathrm{~B}$ & 107.8 \\
\hline $\mathrm{C} 6 \mathrm{~A}-\mathrm{N} 3 \mathrm{~A}-\mathrm{H} 3 \mathrm{~A}$ & 107.4 & $\mathrm{O} 2 \mathrm{~A}-\mathrm{C} 11 \mathrm{~A}-\mathrm{O} 1 \mathrm{~A}$ & $124.3(3)$ \\
\hline $\mathrm{Cr} 1 \mathrm{~A}-\mathrm{N} 3 \mathrm{~A}-\mathrm{H} 3 \mathrm{~A}$ & 107.4 & $\mathrm{O} 2 \mathrm{~A}-\mathrm{C} 11 \mathrm{~A}-\mathrm{C} 12 \mathrm{~A}$ & $121.5(2)$ \\
\hline $\mathrm{C} 7 \mathrm{~A}-\mathrm{N} 4 \mathrm{~A}-\mathrm{C} 8 \mathrm{~A}$ & $112.9(2)$ & $\mathrm{O} 1 \mathrm{~A}-\mathrm{C} 11 \mathrm{~A}-\mathrm{C} 12 \mathrm{~A}$ & $114.15(18)$ \\
\hline $\mathrm{C} 7 \mathrm{~A}-\mathrm{N} 4 \mathrm{~A}-\mathrm{Cr} 1 \mathrm{~A}$ & $108.49(17)$ & $\mathrm{O} 4 \mathrm{~A}-\mathrm{C} 12 \mathrm{~A}-\mathrm{O} 3 \mathrm{~A}$ & $125.2(3)$ \\
\hline $\mathrm{C} 8 \mathrm{~A}-\mathrm{N} 4 \mathrm{~A}-\mathrm{Cr} 1 \mathrm{~A}$ & $117.86(19)$ & $\mathrm{O} 4 \mathrm{~A}-\mathrm{C} 12 \mathrm{~A}-\mathrm{C} 11 \mathrm{~A}$ & $121.2(2)$ \\
\hline $\mathrm{C} 7 \mathrm{~A}-\mathrm{N} 4 \mathrm{~A}-\mathrm{H} 4 \mathrm{~A}$ & 105.5 & $\mathrm{O} 3 \mathrm{~A}-\mathrm{C} 12 \mathrm{~A}-\mathrm{C} 11 \mathrm{~A}$ & $113.63(19)$ \\
\hline
\end{tabular}




\begin{tabular}{|c|c|}
\hline $\mathrm{C} 8 \mathrm{~A}-\mathrm{N} 4 \mathrm{~A}-\mathrm{H} 4 \mathrm{~A}$ & 105.5 \\
\hline $\mathrm{Cr} 1 \mathrm{~A}-\mathrm{N} 4 \mathrm{~A}-\mathrm{H} 4 \mathrm{~A}$ & 105.5 \\
\hline $\mathrm{C} 2 \mathrm{~A}-\mathrm{C} 1 \mathrm{~A}-\mathrm{N} 1 \mathrm{~A}$ & $108.4(2)$ \\
\hline $\mathrm{C} 2 \mathrm{~A}-\mathrm{C} 1 \mathrm{~A}-\mathrm{H} 1 \mathrm{AA}$ & 110.0 \\
\hline $\mathrm{N} 1 \mathrm{~A}-\mathrm{C} 1 \mathrm{~A}-\mathrm{H} 1 \mathrm{AA}$ & 110.0 \\
\hline $\mathrm{C} 2 \mathrm{~A}-\mathrm{C} 1 \mathrm{~A}-\mathrm{H} 1 \mathrm{AB}$ & 110.0 \\
\hline $\mathrm{N} 1 \mathrm{~A}-\mathrm{C} 1 \mathrm{~A}-\mathrm{H} 1 \mathrm{AB}$ & 110.0 \\
\hline $\mathrm{H} 1 \mathrm{AA}-\mathrm{C} 1 \mathrm{~A}-\mathrm{H} 1 \mathrm{AB}$ & 108.4 \\
\hline $\mathrm{N} 2 \mathrm{~A}-\mathrm{C} 2 \mathrm{~A}-\mathrm{C} 1 \mathrm{~A}$ & $109.2(2)$ \\
\hline $\mathrm{N} 2 \mathrm{~A}-\mathrm{C} 2 \mathrm{~A}-\mathrm{H} 2 \mathrm{AA}$ & 109.8 \\
\hline $\mathrm{C} 1 \mathrm{~A}-\mathrm{C} 2 \mathrm{~A}-\mathrm{H} 2 \mathrm{AA}$ & 109.8 \\
\hline $\mathrm{N} 2 \mathrm{~A}-\mathrm{C} 2 \mathrm{~A}-\mathrm{H} 2 \mathrm{AB}$ & 109.8 \\
\hline $\mathrm{C} 1 \mathrm{~A}-\mathrm{C} 2 \mathrm{~A}-\mathrm{H} 2 \mathrm{AB}$ & 109.8 \\
\hline $\mathrm{H} 2 \mathrm{AA}-\mathrm{C} 2 \mathrm{~A}-\mathrm{H} 2 \mathrm{AB}$ & 108.3 \\
\hline $\mathrm{N} 2 \mathrm{~A}-\mathrm{C} 3 \mathrm{~A}-\mathrm{C} 4 \mathrm{~A}$ & $113.8(2)$ \\
\hline $\mathrm{N} 2 \mathrm{~A}-\mathrm{C} 3 \mathrm{~A}-\mathrm{H} 3 \mathrm{AA}$ & 108.8 \\
\hline $\mathrm{C} 4 \mathrm{~A}-\mathrm{C} 3 \mathrm{~A}-\mathrm{H} 3 \mathrm{AA}$ & 108.8 \\
\hline $\mathrm{N} 2 \mathrm{~A}-\mathrm{C} 3 \mathrm{~A}-\mathrm{H} 3 \mathrm{AB}$ & 108.8 \\
\hline $\mathrm{C} 4 \mathrm{~A}-\mathrm{C} 3 \mathrm{~A}-\mathrm{H} 3 \mathrm{AB}$ & 108.8 \\
\hline $\mathrm{H} 3 \mathrm{AA}-\mathrm{C} 3 \mathrm{~A}-\mathrm{H} 3 \mathrm{AB}$ & 107.7 \\
\hline $\mathrm{C} 5 \mathrm{~A}-\mathrm{C} 4 \mathrm{~A}-\mathrm{C} 3 \mathrm{~A}$ & $114.7(3)$ \\
\hline $\mathrm{C} 5 \mathrm{~A}-\mathrm{C} 4 \mathrm{~A}-\mathrm{H} 4 \mathrm{AA}$ & 108.6 \\
\hline $\mathrm{C} 3 \mathrm{~A}-\mathrm{C} 4 \mathrm{~A}-\mathrm{H} 4 \mathrm{AA}$ & 108.6 \\
\hline $\mathrm{C} 5 \mathrm{~A}-\mathrm{C} 4 \mathrm{~A}-\mathrm{H} 4 \mathrm{AB}$ & 108.6 \\
\hline $\mathrm{C} 3 \mathrm{~A}-\mathrm{C} 4 \mathrm{~A}-\mathrm{H} 4 \mathrm{AB}$ & 108.6 \\
\hline $\mathrm{H} 4 \mathrm{AA}-\mathrm{C} 4 \mathrm{~A}-\mathrm{H} 4 \mathrm{AB}$ & 107.6 \\
\hline $\mathrm{N} 3 \mathrm{~A}-\mathrm{C} 5 \mathrm{~A}-\mathrm{C} 4 \mathrm{~A}$ & $112.4(3)$ \\
\hline $\mathrm{N} 3 \mathrm{~A}-\mathrm{C} 5 \mathrm{~A}-\mathrm{H} 5 \mathrm{AA}$ & 109.1 \\
\hline $\mathrm{C} 4 \mathrm{~A}-\mathrm{C} 5 \mathrm{~A}-\mathrm{H} 5 \mathrm{AA}$ & 109.1 \\
\hline $\mathrm{C} 10 \mathrm{~A}-\mathrm{N} 1 \mathrm{~A}-\mathrm{C} 1 \mathrm{~A}-\mathrm{C} 2 \mathrm{~A}$ & $174.0(2)$ \\
\hline $\mathrm{Cr} 1 \mathrm{~A}-\mathrm{N} 1 \mathrm{~A}-\mathrm{C} 1 \mathrm{~A}-\mathrm{C} 2 \mathrm{~A}$ & $47.1(2)$ \\
\hline $\mathrm{C} 3 \mathrm{~A}-\mathrm{N} 2 \mathrm{~A}-\mathrm{C} 2 \mathrm{~A}-\mathrm{C} 1 \mathrm{~A}$ & $166.9(3)$ \\
\hline $\mathrm{Cr} 1 \mathrm{~A}-\mathrm{N} 2 \mathrm{~A}-\mathrm{C} 2 \mathrm{~A}-\mathrm{C} 1 \mathrm{~A}$ & $33.3(3)$ \\
\hline $\mathrm{N} 1 \mathrm{~A}-\mathrm{C} 1 \mathrm{~A}-\mathrm{C} 2 \mathrm{~A}-\mathrm{N} 2 \mathrm{~A}$ & $-54.5(3)$ \\
\hline $\mathrm{C} 2 \mathrm{~A}-\mathrm{N} 2 \mathrm{~A}-\mathrm{C} 3 \mathrm{~A}-\mathrm{C} 4 \mathrm{~A}$ & $-75.2(3)$ \\
\hline $\mathrm{Cr} 1 \mathrm{~A}-\mathrm{N} 2 \mathrm{~A}-\mathrm{C} 3 \mathrm{~A}-\mathrm{C} 4 \mathrm{~A}$ & $53.4(3)$ \\
\hline $\mathrm{N} 2 \mathrm{~A}-\mathrm{C} 3 \mathrm{~A}-\mathrm{C} 4 \mathrm{~A}-\mathrm{C} 5 \mathrm{~A}$ & $-66.3(4)$ \\
\hline $\mathrm{C} 6 \mathrm{~A}-\mathrm{N} 3 \mathrm{~A}-\mathrm{C} 5 \mathrm{~A}-\mathrm{C} 4 \mathrm{~A}$ & $177.8(2)$ \\
\hline $\mathrm{Cr} 1 \mathrm{~A}-\mathrm{N} 3 \mathrm{~A}-\mathrm{C} 5 \mathrm{~A}-\mathrm{C} 4 \mathrm{~A}$ & $-60.4(3)$ \\
\hline $\mathrm{C} 3 \mathrm{~A}-\mathrm{C} 4 \mathrm{~A}-\mathrm{C} 5 \mathrm{~A}-\mathrm{N} 3 \mathrm{~A}$ & $69.8(4)$ \\
\hline $\mathrm{C} 5 \mathrm{~A}-\mathrm{N} 3 \mathrm{~A}-\mathrm{C} 6 \mathrm{~A}-\mathrm{C} 7 \mathrm{~A}$ & $175.0(2)$ \\
\hline $\mathrm{Cr} 1 \mathrm{~A}-\mathrm{N} 3 \mathrm{~A}-\mathrm{C} 6 \mathrm{~A}-\mathrm{C} 7 \mathrm{~A}$ & $46.5(3)$ \\
\hline $\mathrm{C} 8 \mathrm{~A}-\mathrm{N} 4 \mathrm{~A}-\mathrm{C} 7 \mathrm{~A}-\mathrm{C} 6 \mathrm{~A}$ & $168.8(3)$ \\
\hline $\mathrm{Cr} 1 \mathrm{~A}-\mathrm{N} 4 \mathrm{~A}-\mathrm{C} 7 \mathrm{~A}-\mathrm{C} 6 \mathrm{~A}$ & $36.2(3)$ \\
\hline $\mathrm{N} 3 \mathrm{~A}-\mathrm{C} 6 \mathrm{~A}-\mathrm{C} 7 \mathrm{~A}-\mathrm{N} 4 \mathrm{~A}$ & $-55.8(3)$ \\
\hline $\mathrm{C} 7 \mathrm{~A}-\mathrm{N} 4 \mathrm{~A}-\mathrm{C} 8 \mathrm{~A}-\mathrm{C} 9 \mathrm{~A}$ & $-72.7(4)$ \\
\hline $\mathrm{Cr} 1 \mathrm{~A}-\mathrm{N} 4 \mathrm{~A}-\mathrm{C} 8 \mathrm{~A}-\mathrm{C} 9 \mathrm{~A}$ & $55.1(3)$ \\
\hline
\end{tabular}

\begin{tabular}{|c|c|}
\hline $\mathrm{O} 3 \mathrm{~B} 2-\mathrm{Cr} 2 \mathrm{~B}-\mathrm{O} 4 \mathrm{~B} 2$ & $118.5(8)$ \\
\hline $\mathrm{O} 3 \mathrm{~B} 2-\mathrm{Cr} 2 \mathrm{~B}-\mathrm{O} 2 \mathrm{~B} 2$ & $111.1(5)$ \\
\hline $\mathrm{O} 4 \mathrm{~B} 2-\mathrm{Cr} 2 \mathrm{~B}-\mathrm{O} 2 \mathrm{~B} 2$ & $104.7(8)$ \\
\hline $\mathrm{O} 2 \mathrm{~B} 1-\mathrm{Cr} 2 \mathrm{~B}-\mathrm{O} 3 \mathrm{~B} 1$ & $106.5(6)$ \\
\hline $\mathrm{O} 2 \mathrm{~B} 1-\mathrm{Cr} 2 \mathrm{~B}-\mathrm{O} 4 \mathrm{~B} 1$ & $106.1(6)$ \\
\hline $\mathrm{O} 3 \mathrm{~B} 1-\mathrm{Cr} 2 \mathrm{~B}-\mathrm{O} 4 \mathrm{~B} 1$ & $103.8(3)$ \\
\hline $\mathrm{O} 3 \mathrm{~B} 2-\mathrm{Cr} 2 \mathrm{~B}-\mathrm{O} 1 \mathrm{~B}$ & $112.5(4)$ \\
\hline $\mathrm{O} 4 \mathrm{~B} 2-\mathrm{Cr} 2 \mathrm{~B}-\mathrm{O} 1 \mathrm{~B}$ & $128.2(8)$ \\
\hline $\mathrm{O} 2 \mathrm{~B} 1-\mathrm{Cr} 2 \mathrm{~B}-\mathrm{O} 1 \mathrm{~B}$ & $115.3(6)$ \\
\hline $\mathrm{O} 2 \mathrm{~B} 2-\mathrm{Cr} 2 \mathrm{~B}-\mathrm{O} 1 \mathrm{~B}$ & $62.6(3)$ \\
\hline $\mathrm{O} 3 \mathrm{~B} 1-\mathrm{Cr} 2 \mathrm{~B}-\mathrm{O} 1 \mathrm{~B}$ & $117.0(3)$ \\
\hline $\mathrm{O} 4 \mathrm{~B} 1-\mathrm{Cr} 2 \mathrm{~B}-\mathrm{O} 1 \mathrm{~B}$ & $107.1(3)$ \\
\hline $\mathrm{O} 3 \mathrm{~B} 2-\mathrm{Cr} 2 \mathrm{~B}-\mathrm{O} 1 \mathrm{~B}^{\mathrm{i}}$ & $109.3(4)$ \\
\hline $\mathrm{O} 4 \mathrm{~B} 2-\mathrm{Cr} 2 \mathrm{~B}-\mathrm{O} 1 \mathrm{~B}^{\mathrm{i}}$ & $107.5(8)$ \\
\hline $\mathrm{O} 2 \mathrm{~B} 1-\mathrm{Cr} 2 \mathrm{~B}-\mathrm{O} 1 \mathrm{~B}^{\mathrm{i}}$ & $100.0(6)$ \\
\hline $\mathrm{O} 2 \mathrm{~B} 2-\mathrm{Cr} 2 \mathrm{~B}-\mathrm{O} 1 \mathrm{~B}^{\mathrm{i}}$ & $104.8(3)$ \\
\hline $\mathrm{O} 3 \mathrm{~B} 1-\mathrm{Cr} 2 \mathrm{~B}-\mathrm{O} 1 \mathrm{~B}^{\mathrm{i}}$ & $85.3(3)$ \\
\hline $\mathrm{O} 4 \mathrm{~B} 1-\mathrm{Cr} 2 \mathrm{~B}-\mathrm{O} 1 \mathrm{~B}^{\mathrm{i}}$ & $148.4(2)$ \\
\hline $\mathrm{O} 1 \mathrm{~B}-\mathrm{Cr} 2 \mathrm{~B}-\mathrm{O} 1 \mathrm{~B}^{\mathrm{i}}$ & $44.0(3)$ \\
\hline $\mathrm{O} 1 \mathrm{~B}-\mathrm{O} 1 \mathrm{~B}-\mathrm{Cr} 2 \mathrm{~B}$ & $74.5(3)$ \\
\hline $\mathrm{O} 1 \mathrm{~B}^{\mathrm{i}}-\mathrm{O} 1 \mathrm{~B}-\mathrm{O} 2 \mathrm{~B} 2$ & $128.7(5)$ \\
\hline $\mathrm{Cr} 2 \mathrm{~B}-\mathrm{O} 1 \mathrm{~B}-\mathrm{O} 2 \mathrm{~B} 2$ & $57.1(3)$ \\
\hline $\mathrm{O} 1 \mathrm{~B}-\mathrm{O} 1 \mathrm{~B}-\mathrm{Cr} 2 \mathrm{~B}^{\mathrm{i}}$ & $61.5(3)$ \\
\hline $\mathrm{Cr} 2 \mathrm{~B}-\mathrm{O} 1 \mathrm{~B}-\mathrm{Cr} 2 \mathrm{~B}^{\mathrm{i}}$ & $136.0(3)$ \\
\hline $\mathrm{Cr} 2 \mathrm{~B}-\mathrm{O} 2 \mathrm{~B} 2-\mathrm{O} 1 \mathrm{~B}$ & $60.2(3)$ \\
\hline $\mathrm{H} 1 \mathrm{O} 1-\mathrm{O} 1 \mathrm{~S}-\mathrm{H} 2 \mathrm{O} 1$ & $104(2)$ \\
\hline $\mathrm{H} 1 \mathrm{O} 2-\mathrm{O} 2 \mathrm{~S}-\mathrm{H} 2 \mathrm{O} 2$ & $122(3)$ \\
\hline $\mathrm{H} 1 \mathrm{O} 3-\mathrm{O} 3 \mathrm{~S}-\mathrm{H} 2 \mathrm{O} 3$ & $105(2)$ \\
\hline $\mathrm{H} 1 \mathrm{O} 4-\mathrm{O} 4 \mathrm{~S}-\mathrm{H} 2 \mathrm{O} 4$ & $114(3)$ \\
\hline $\mathrm{O} 2 \mathrm{~A}-\mathrm{C} 11 \mathrm{~A}-\mathrm{C} 12 \mathrm{~A}-\mathrm{O} 4 \mathrm{~A}$ & $-1.2(4)$ \\
\hline $\mathrm{O} 1 \mathrm{~A}-\mathrm{C} 11 \mathrm{~A}-\mathrm{C} 12 \mathrm{~A}-\mathrm{O} 4 \mathrm{~A}$ & $178.8(2)$ \\
\hline $\mathrm{O} 2 \mathrm{~A}-\mathrm{C} 11 \mathrm{~A}-\mathrm{C} 12 \mathrm{~A}-\mathrm{O} 3 \mathrm{~A}$ & $178.4(2)$ \\
\hline $\mathrm{O} 1 \mathrm{~A}-\mathrm{C} 11 \mathrm{~A}-\mathrm{C} 12 \mathrm{~A}-\mathrm{O} 3 \mathrm{~A}$ & $-1.6(3)$ \\
\hline $\mathrm{O} 3 \mathrm{~B} 2-\mathrm{Cr} 2 \mathrm{~B}-\mathrm{O} 1 \mathrm{~B}-\mathrm{O} 1 \mathrm{~B}^{\mathrm{i}}$ & $-95.0(7)$ \\
\hline $\mathrm{O} 4 \mathrm{~B} 2-\mathrm{Cr} 2 \mathrm{~B}-\mathrm{O} 1 \mathrm{~B}-\mathrm{O} 1 \mathrm{~B}^{\mathrm{i}}$ & $74.6(12)$ \\
\hline $\mathrm{O} 2 \mathrm{~B} 1-\mathrm{Cr} 2 \mathrm{~B}-\mathrm{O} 1 \mathrm{~B}-\mathrm{O} 1 \mathrm{~B}^{\mathrm{i}}$ & $77.7(9)$ \\
\hline $\mathrm{O} 2 \mathrm{~B} 2-\mathrm{Cr} 2 \mathrm{~B}-\mathrm{O} 1 \mathrm{~B}-\mathrm{O} 1 \mathrm{~B}^{\mathrm{i}}$ & $162.1(8)$ \\
\hline $\mathrm{O} 3 \mathrm{~B} 1-\mathrm{Cr} 2 \mathrm{~B}-\mathrm{O} 1 \mathrm{~B}-\mathrm{O} 1 \mathrm{~B}^{\mathrm{i}}$ & $-48.7(7)$ \\
\hline $\mathrm{O} 4 \mathrm{~B} 1-\mathrm{Cr} 2 \mathrm{~B}-\mathrm{O} 1 \mathrm{~B}-\mathrm{O} 1 \mathrm{~B}^{\mathrm{i}}$ & $-164.6(5)$ \\
\hline $\mathrm{O} 3 \mathrm{~B} 2-\mathrm{Cr} 2 \mathrm{~B}-\mathrm{O} 1 \mathrm{~B}-\mathrm{O} 2 \mathrm{~B} 2$ & $103.0(6)$ \\
\hline $\mathrm{O} 4 \mathrm{~B} 2-\mathrm{Cr} 2 \mathrm{~B}-\mathrm{O} 1 \mathrm{~B}-\mathrm{O} 2 \mathrm{~B} 2$ & $-87.4(11)$ \\
\hline $\mathrm{O} 1 \mathrm{~B}^{\mathrm{i}}-\mathrm{Cr} 2 \mathrm{~B}-\mathrm{O} 1 \mathrm{~B}-\mathrm{O} 2 \mathrm{~B} 2$ & $-162.1(8)$ \\
\hline $\mathrm{O} 3 \mathrm{~B} 2-\mathrm{Cr} 2 \mathrm{~B}-\mathrm{O} 1 \mathrm{~B}-\mathrm{Cr} 2 \mathrm{~B}^{\mathrm{i}}$ & $-95.0(7)$ \\
\hline $\mathrm{O} 4 \mathrm{~B} 2-\mathrm{Cr} 2 \mathrm{~B}-\mathrm{O} 1 \mathrm{~B}-\mathrm{Cr} 2 \mathrm{~B}^{\mathrm{i}}$ & $74.6(12)$ \\
\hline $\mathrm{O} 2 \mathrm{~B} 1-\mathrm{Cr} 2 \mathrm{~B}-\mathrm{O} 1 \mathrm{~B}-\mathrm{Cr} 2 \mathrm{~B}^{\mathrm{i}}$ & $77.7(9)$ \\
\hline $\mathrm{O} 2 \mathrm{~B} 2-\mathrm{Cr} 2 \mathrm{~B}-\mathrm{O} 1 \mathrm{~B}-\mathrm{Cr} 2 \mathrm{~B}^{\mathrm{i}}$ & $162.1(8)$ \\
\hline $\mathrm{O} 3 \mathrm{~B} 1-\mathrm{Cr} 2 \mathrm{~B}-\mathrm{O} 1 \mathrm{~B}-\mathrm{Cr} 2 \mathrm{~B}^{\mathrm{i}}$ & $-48.7(7)$ \\
\hline
\end{tabular}




$\begin{array}{llll}\mathrm{N} 4 \mathrm{~A}-\mathrm{C} 8 \mathrm{~A}-\mathrm{C} 9 \mathrm{~A}-\mathrm{C} 10 \mathrm{~A} & -67.0(4) & \mathrm{O} 4 \mathrm{~B} 1-\mathrm{Cr} 2 \mathrm{~B}-\mathrm{O} 1 \mathrm{~B}-\mathrm{Cr} 2 \mathrm{~B}^{\mathrm{i}} & -164.6(5) \\ \mathrm{C} 1 \mathrm{~A}-\mathrm{N} 1 \mathrm{~A}-\mathrm{C} 10 \mathrm{~A}-\mathrm{C} 9 \mathrm{~A} & 179.0(2) & \mathrm{O} 1 \mathrm{~B}-\mathrm{Cr} 2 \mathrm{~B}-\mathrm{O} 1 \mathrm{~B}-\mathrm{Cr} 2 \mathrm{~B}^{\mathrm{i}} & 0.005(1) \\ \mathrm{C} 1 \mathrm{~A}-\mathrm{N} 1 \mathrm{~A}-\mathrm{C} 10 \mathrm{~A}-\mathrm{C} 9 \mathrm{~A} & -61.1(3) & \mathrm{O} 3 \mathrm{~B} 2-\mathrm{Cr} 2 \mathrm{~B}-\mathrm{O} 2 \mathrm{~B} 2-\mathrm{O} 1 \mathrm{~B} & -105.2(5) \\ \mathrm{C} 8 \mathrm{~A}-\mathrm{C} 9 \mathrm{~A}-\mathrm{C} 10 \mathrm{~A}-\mathrm{N} 1 \mathrm{~A} & 70.3(4) & \mathrm{O} 4 \mathrm{~B} 2-\mathrm{Cr} 2 \mathrm{~B}-\mathrm{O} 2 \mathrm{~B} 2-\mathrm{O} 1 \mathrm{~B} & 125.8(8) \\ \mathrm{Cr} 1 \mathrm{~A}-\mathrm{O} 1 \mathrm{~A}-\mathrm{C} 11 \mathrm{~A}-\mathrm{O} 2 \mathrm{~A} & -178.9(2) & \mathrm{O} 1 \mathrm{~B}-\mathrm{Cr} 2 \mathrm{~B}-\mathrm{O} 2 \mathrm{~B} 2-\mathrm{O} 1 \mathrm{~B} & 12.8(5) \\ \text { Cr1A-O1A-C11A-C12A } & 1.1(3) & \mathrm{O} 1 \mathrm{~B}-\mathrm{O} 1 \mathrm{~B}-\mathrm{O} 2 \mathrm{~B} 2-\mathrm{Cr} 2 \mathrm{~B} & -22.3(10) \\ \text { Cr1A-O3A-C12A-O4A } & -179.2(2) & \mathrm{Cr} 2 \mathrm{~B}-\mathrm{O} 1 \mathrm{~B}-\mathrm{O} 2 \mathrm{~B} 2-\mathrm{Cr} 2 \mathrm{~B} & -138.9(13) \\ \text { Cr1A-O3A-C12A-C11A } & 1.2(3) & & \end{array}$

Symmetry code: (i) $-x+2,-y,-z+1$.

Hydrogen-bond geometry $\left(A,{ }^{\circ}\right)$

\begin{tabular}{lllll}
\hline$D-\mathrm{H} \cdots A$ & $D-\mathrm{H}$ & $\mathrm{H} \cdots A$ & $D \cdots A$ & $D-\mathrm{H} \cdots A$ \\
\hline $\mathrm{N} 1 A-\mathrm{H} 1 A \cdots \mathrm{O} 4 A^{\mathrm{ii}}$ & 0.98 & 1.99 & $2.804(3)$ & 139 \\
$\mathrm{~N} 2 A-\mathrm{H} 2 A \cdots \mathrm{O} 1 S$ & 0.98 & 2.00 & $2.894(3)$ & 150 \\
$\mathrm{~N} 3 A-\mathrm{H} 3 A \cdots \mathrm{O} 2 A^{\mathrm{ii}}$ & 0.98 & 1.89 & $2.842(3)$ & 163 \\
$\mathrm{~N} 4 A-\mathrm{H} 4 A \cdots \mathrm{O} 2 B 1^{\mathrm{iii}}$ & 0.98 & 2.25 & $3.100(16)$ & 144 \\
$\mathrm{~N} 4 A-\mathrm{H} 4 A \cdots \mathrm{O} 4 B 1^{\mathrm{iii}}$ & 0.98 & 2.37 & $3.108(6)$ & 132 \\
$\mathrm{~N} 4 A-\mathrm{H} 4 A \cdots \mathrm{O} 4 B 2^{\mathrm{iii}}$ & 0.98 & 2.15 & $3.05(2)$ & 151 \\
$\mathrm{O} 1 S-\mathrm{H} 1 O 1 \cdots \mathrm{O} 4 B 1$ & $0.85(1)$ & $2.33(5)$ & $2.876(6)$ & $123(5)$ \\
$\mathrm{O} 1 S-\mathrm{H} 1 O 1 \cdots \mathrm{O} 3 B 2$ & $0.85(1)$ & $2.20(4)$ & $2.903(10)$ & $141(5)$ \\
$\mathrm{O} 2 S-\mathrm{H} 2 O 2 \cdots \mathrm{O} 3 S$ & $0.85(1)$ & $1.91(2)$ & $2.729(6)$ & $164(6)$ \\
$\mathrm{O} 3 S-\mathrm{H} 1 O 3 \cdots \mathrm{O} 3 B 1$ & $0.85(1)$ & $1.93(3)$ & $2.725(6)$ & $156(7)$ \\
$\mathrm{O} 3 S-\mathrm{H} 1 O 3 \cdots \mathrm{O} 3 B 2$ & $0.85(1)$ & $2.28(2)$ & $3.113(10)$ & $167(6)$ \\
$\mathrm{O} 3 S-\mathrm{H} 2 O 3 \cdots \mathrm{O} 1 A^{\mathrm{iv}}$ & $0.85(1)$ & $2.52(3)$ & $3.301(4)$ & $154(6)$ \\
$\mathrm{O} 3 S-\mathrm{H} 2 O 3 \cdots \mathrm{O} 2 A^{\mathrm{iv}}$ & $0.85(1)$ & $2.21(5)$ & $2.911(5)$ & $140(6)$ \\
$\mathrm{O} 4 S-\mathrm{H} 1 O 4 \cdots \mathrm{O} 4 A$ & $0.84(1)$ & $2.11(4)$ & $2.834(4)$ & $144(6)$ \\
$\mathrm{O} 4 S-\mathrm{H} 2 O 4 \cdots \mathrm{O} 2 S$ & $0.85(1)$ & $1.89(2)$ & $2.723(5)$ & $166(6)$ \\
& & & &
\end{tabular}

Symmetry codes: (ii) $x-1, y, z$; (iii) $x,-y+1 / 2, z+1 / 2$; (iv) $x,-y+1 / 2, z-1 / 2$. 